\title{
A Functionally-Fitted Block Numerov Method for Solving Second-Order Initial-Value Problems with Oscillatory Solutions
}

\author{
R. I. Abdulganiy (1), Higinio Ramos (1), O. A. Akinfenwa(1) and \\ S. A. Okunuga(i)
}

\begin{abstract}
A functionally-fitted Numerov-type method is developed for the numerical solution of second-order initial-value problems with oscillatory solutions. The basis functions are considered among trigonometric and hyperbolic ones. The characteristics of the method are studied, particularly, it is shown that it has a third order of convergence for the general second-order ordinary differential equation, $y^{\prime \prime}=f\left(x, y, y^{\prime}\right)$, it is a fourth order convergent method for the special second-order ordinary differential equation, $y^{\prime \prime}=f(x, y)$. Comparison with other methods in the literature, even of higher order, shows the good performance of the proposed method.
\end{abstract}

Mathematics Subject Classification. Primary 65L05; Secondary 65L20.

Keywords. Block method, convergence analysis, functionally-fitted approach, numerov-type method, trigonometric functions, hyperbolic functions.

\section{Introduction}

Conventionally, second-order differential equations (DEs) whether solving analytically or numerically, can be reduced into an equivalent system of firstorder equations to leverage on methods constructed for first-order systems (see Enright [7], Lambert [28], Brugnano et al. [5] and Fatunla [12] for a numerical point of view). According to Onumanyi et al. [31] and Awoyemi [4], the approach of reducing a second-order differential equation to a system of first-order differential equations is marred by a large human effort and a more demanding storage memory during implementation. It is even more complex when writing a computer code for the method, particularly the subroutine needed to supply starting values required for such method, due to the 
large dimension of the resulting first-order system. Twizell and Khaliq [42], Coleman and Duxbury [6], Hairer and his collaborators [18,19], and Lambert and Watson [29], established that solving second-order Differential Equations directly saves about half of the storage space. Hence, the direct solution of second-order DEs is more preferable.

In what follows we will consider a Functionally-Fitted Block Numerov's Method (FFBNM) for the integration of second-order Initial-Value Problem (IVP) systems of the form

$$
y^{\prime \prime}=f\left(x, y, y^{\prime}\right), y\left(x_{0}\right)=y_{0}, y^{\prime}\left(x_{0}\right)=y_{0}^{\prime},
$$

whose solution is assumed to be oscillatory or periodic, and the frequency is approximately known in advance, with $f: \mathbb{R} \times \mathbb{R}^{m} \times \mathbb{R}^{m} \rightarrow \mathbb{R}^{m}$ a smooth function that satisfies a Lipschitz condition, being $m$ the dimension of the system.

As noticed before, solving the problem in (1.1) directly is preferable, since about half of the storage space can be saved, especially, if the dimension of the system is large (see Coleman and Duxbury [6]).

Many prominent authors including Simos [39], Coleman and Duxbury [6], Achar [3], Franco [14-17], Wang et al. [46], Van Daele et al. [43], Wang et al. [47,48], Fang et al. [8-11,49], Tsitouras [41], Li et al. [30], Jator et al. [21-27], Ramos et al. [32-38,40], among others, have presented numerical methods for directly solving the equation in (1.1) without transforming it into an equivalent system of first-order ODEs. However, the above contributions did not consider that the approximate interpolating function was a linear combination of monomial, trigonometric terms and hyperbolic terms. We emphasize that we will introduce a method which approximates the solution of the IVP in (1.1) considering that the method is exact when the solution is in the space generated by $\sigma(x)=\{1, \sin (\omega x), \cos (\omega x), \sinh (\omega x), \cosh (\omega x)\}$. This functional basis is motivated by its easiness to analyze and its expectations to provide improved approximations for second-order initial-value problems with periodic or oscillatory solutions.

The rest of the paper is organized as follows: the theoretical and basic elements of the FFBNM are discussed in Sect. 2. The basic properties of the FFBNM are studied in Sect. 3. Some numerical examples are considered in Sect. 4 to illustrate the performance of the method, and finally, Sect. 5 puts and end to the paper with some conclusions.

\section{Development of the FFBNM}

In this section, we develop a Continuous Block Method of Numerov type (CBNM) on the interval $\left[x_{n}, x_{n+2}\right]$ to produce a discrete Block Numerov Method. To do this we consider $m=1$, that is, the scalar case, although, as can be seen in the numerical section, the method can be applied in a component-wise formulation for solving differential systems. Three complementary formulas as a by-product via the CBNM formula to approximate the first derivatives are generated too, to form the Block Numerov Method. The CBNM has the general form 


$$
\tau(x)=\sum_{j=0}^{1} \alpha_{j}(x, u) y_{n+j}+h^{2} \sum_{j=0}^{2} \beta_{j}(x, u) f_{n+j},
$$

where $u=\omega h$, and $\alpha_{j}, \beta_{j}$ are coefficients to be determined uniquely, that depend on the parameter frequency $\omega$ and the step size $h$. As usual, $y_{n+j}, y_{n+j}^{\prime}$ are numerical approximations to the exact values $y\left(x_{n+j}\right), y^{\prime}\left(x_{n+j}\right)$, and $f_{n+j}=f\left(x_{n+j}, y_{n+j}, y_{n+j}^{\prime}\right)$.

We consider that the true solution $y(x)$ is locally approximated on the block interval $\left[x_{n}, x_{n+2}\right]$ by a solution $\tau(x)$ of the form

$$
\tau(x)=a_{0}+a_{1} \sin (\omega x)+a_{2} \cos (\omega x)+a_{3} \sinh (\omega x)+a_{4} \cosh (\omega x),
$$

where the coefficients $a_{i}$ will be obtained demanding that the following system of equations is satisfied

$$
\left\{\begin{array}{l}
\tau\left(x_{n+j}\right)=y_{n+j}, \quad j=0,1, \\
\tau^{\prime \prime}\left(x_{n+j}\right)=f_{n+j}, \quad j=0,1,2 .
\end{array}\right.
$$

Now we state the main result that aids the construction of the CBNMs as follows:

Theorem 2.1. Let $\tau(x)$ be the function given in (2.2) which satisfies the system in (2.3). The continuous approximation that will be used to obtain the FFBNM is given by

$$
\tau(x)=\Theta^{T}\left(\varsigma^{-1}\right)^{T}\left[\left(\Omega^{-1}\right)^{T} \sigma(x)\right],
$$

where $\varsigma$ and $\Omega$ are $5 \times 5$ non-singular lower and upper triangular matrices given by

$$
\varsigma=\left[\begin{array}{ccccc}
1 & 0 & 0 & 0 & 0 \\
\varsigma_{2,1} & 1 & 0 & 0 & 0 \\
\varsigma_{3,1} & \varsigma_{3,2} & 1 & 0 & 0 \\
\varsigma_{4,1} & \varsigma_{4,2} & \varsigma_{4,3} & 1 & 0 \\
\varsigma_{5,1} & \varsigma_{5,2} & \varsigma_{5,3} & \varsigma_{5,4} & 1
\end{array}\right], \quad \Omega=\left[\begin{array}{ccccc}
\Omega_{1,1} & \Omega_{1,2} & \Omega_{1,3} & \Omega_{1,4} & \Omega_{1,5} \\
0 & \Omega_{2,2} & \Omega_{2,3} & \Omega_{2,4} & \Omega_{2,5} \\
0 & 0 & \Omega_{3,3} & \Omega_{3,4} & \Omega_{3,5} \\
0 & 0 & 0 & \Omega_{4,4} & \Omega_{4,5} \\
0 & 0 & 0 & 0 & \Omega_{5,5}
\end{array}\right],
$$

$\sigma(x)$ and $\Theta$ are vectors defined by $\sigma(x)=\left(\sigma_{0}(x), \sigma_{1}(x), \sigma_{2}(x), \sigma_{3}(x), \sigma_{4}(x)\right)^{T}$, with $\left\{\sigma_{j}(x)\right\}_{j=0}^{4}=\{1, \sin (\omega x), \cos (\omega x), \sinh (\omega x), \cosh (\omega x)\}$ and $\Theta=\left(y_{n}, y_{n+1}, f_{n}, f_{n+1}, f_{n+2}\right)^{T}$, respectively (the superscript $T$ denotes the transpose).

Proof. Define the following non-singular matrix as the matrix containing the evaluation of the basis functions at the grid points,

$$
V=\left[\begin{array}{ccccc}
\sigma_{0}\left(x_{n}\right) & \sigma_{1}\left(x_{n}\right) & \sigma_{2}\left(x_{n}\right) & \sigma_{3}\left(x_{n}\right) & \sigma_{4}\left(x_{n}\right) \\
\sigma_{0}\left(x_{n+1}\right) & \sigma_{1}\left(x_{n+1}\right) & \sigma_{2}\left(x_{n+1}\right) & \sigma_{3}\left(x_{n+1}\right) & \sigma_{4}\left(x_{n+1}\right) \\
\sigma_{0}^{\prime \prime}\left(x_{n}\right) & \sigma_{1}^{\prime \prime}\left(x_{n}\right) & \sigma_{2}^{\prime \prime}\left(x_{n}\right) & \sigma_{3}^{\prime \prime}\left(x_{n}\right) & \sigma_{4}^{\prime \prime}\left(x_{n}\right) \\
\sigma_{0}^{\prime \prime}\left(x_{n+1}\right) & \sigma_{1}^{\prime \prime}\left(x_{n+1}\right) & \sigma_{2}^{\prime \prime}\left(x_{n+1}\right) & \sigma_{3}^{\prime \prime}\left(x_{n+1}\right) & \sigma_{4}^{\prime \prime}\left(x_{n+1}\right) \\
\sigma_{0}^{\prime \prime}\left(x_{n+2}\right) & \sigma_{1}^{\prime \prime}\left(x_{n+2}\right) & \sigma_{2}^{\prime \prime}\left(x_{n+2}\right) & \sigma_{3}^{\prime \prime}\left(x_{n+2}\right) & \sigma_{4}^{\prime \prime}\left(x_{n+2}\right)
\end{array}\right] .
$$


To solve the system of equations in (2.3), we require that the coefficients in (2.1) are expressed in terms of the assumed basis functions as follows

$$
\begin{aligned}
& \alpha_{j}(x, u)=\sum_{i=0}^{4} \alpha_{i, j}(x, u) \sigma_{i}(x), \quad j=0,1 . \\
& \beta_{j}(x, u)=\sum_{i=0}^{4} \beta_{i, j}(x, u) \sigma_{i}(x), \quad j=0,1,2 .
\end{aligned}
$$

Substituting equations (2.5) and (2.6) into the equation (2.1) yields

$$
\tau(x)=\sum_{i=0}^{4}\left\{\sum_{j=0}^{1} \alpha_{i, j}(x, u) y_{n+j}+h^{2} \sum_{j=0}^{2} \beta_{i, j}(x, u) f_{n+j}\right\} \sigma_{i}(x) .
$$

Letting

$$
\Delta_{i}=\sum_{j=0}^{1} \alpha_{i, j}(x, u) y_{n+j}+h^{2} \sum_{j=0}^{2} \beta_{i, j}(x, u) f_{n+j}, \quad i=0(1) 4,
$$

equation (2.7) becomes

$$
\tau(x)=\sum_{i=0}^{4} \Delta_{i} \sigma_{i}(x)=\Delta^{T} \sigma(x),
$$

where $\Delta=\left(\Delta_{0}, \Delta_{1}, \Delta_{2}, \Delta_{3}, \Delta_{4}\right)^{T}$ is a vector of undetermined coefficients.

Imposing the conditions in equation (2.3) on equation (2.8), a system of five equations expressed as $V \Delta=\Theta$ is obtained, being the undetermined coefficients ascertained simultaneously through the LU decomposition method. Factorizing $V$ into a product of a non-singular lower matrix, $\varsigma$, and a nonsingular upper triangular one, $\Omega$, and using the LU decomposition method we obtain

$$
\Delta=\Omega^{-1}\left(\varsigma^{-1} \Theta\right)
$$

It follows from (2.8) that

$$
\tau(x)=\left(\Omega^{-1}\left(\varsigma^{-1} \Theta\right)\right)^{T} \sigma(x)=\Theta^{T}\left(\varsigma^{-1}\right)^{T}\left[\left(\Omega^{-1}\right)^{T} \sigma(x)\right],
$$

which is the desired result in (2.4).

Remark 2.2. The specific form of matrices $\varsigma, \Omega$ and $V$ is provided in the Appendix.

Remark 2.3. We emphasize that equation (2.4) is of the form presented in equation (2.1), which together with its first derivative provide the main formula and three complementary formulas of the Functionally-Fitted Block Numerov Method (FFBNM). 


\subsection{Specification of FFBNM}

The main formula and the three complementary formulas that form the block method FFBNM are obtained by evaluating the equation in (2.4) at $x=x_{n+2}$, and its first derivative at the set of points $\left\{x_{n}, x_{n+1}, x_{n+2}\right\}$. The resulting formulas are given by

$$
\begin{gathered}
h y_{n}^{\prime}=\alpha_{0,3} y_{n}+\alpha_{1,3} y_{n+1}+h^{2}\left(\beta_{0,3}(u) f_{n}+\beta_{1,3}(u) f_{n+1}+\beta_{2,3}(u) f_{n+2}\right), \\
h y_{n+1}^{\prime}=\alpha_{0,2} y_{n}+\alpha_{1,2} y_{n+1}+h^{2}\left(\beta_{0,2}(u) f_{n}+\beta_{1,2}(u) f_{n+1}+\beta_{2,2}(u) f_{n+2}\right), \\
h y_{n+2}^{\prime}=\alpha_{0,1} y_{n}+\alpha_{1,1} y_{n+1}+h^{2}\left(\beta_{0,1}(u) f_{n}+\beta_{1,1}(u) f_{n+1}+\beta_{2,1}(u) f_{n+2}\right), \\
y_{n+2}-2 y_{n+1}+y_{n}=h^{2}\left(\beta_{0}(u) f_{n}+\beta_{1}(u) f_{n+1}+\beta_{2}(u) f_{n+2}\right) .
\end{gathered}
$$

The coefficients of these formulas are as follow:

$$
\begin{aligned}
& \alpha_{1,3}=-\alpha_{0,3}=\frac{u}{2}(\cot (u)+\operatorname{coth}(u)) \\
& \beta_{0,3}=\frac{b_{03}}{2 u \sinh (u)(-\cosh (u)+\cos (u)) \sin (u)} \\
& \beta_{1,3}=\frac{b_{13}}{2 u \sinh (u)(-\cosh (u)+\cos (u)) \sin (u)} \\
& \beta_{2,3}=\frac{(-\cos (u)+1) \sinh (u)-\sin (u)(\cosh (u)-1)}{u(\sin (u) \sinh (2 u)-\sinh (u) \sin (2 u))}
\end{aligned}
$$

with

$$
\begin{aligned}
b_{03}= & (-\sinh (u) \cos (u)-\sin (u)(\cos (u)-1)) \cosh (u)-\sin (u) \\
& +\left((\cos (u))^{2}+\cos (u)-1\right) \sinh (u)+\sin (u)(\cosh (u))^{2}, \\
b_{13}= & ((-\sin (u)-\sinh (u)) \cos (u)+2 \sinh (u)) \cosh (u) \\
& -\sinh (u)(\cos (u))^{2}+2 \sin (u) \cos (u)-\sin (u)(\cosh (u))^{2} . \\
\alpha_{1,2}= & -\alpha_{0,2}=\frac{u}{2}(\csc (u)+\operatorname{csch}(u)) \\
\beta_{0,2}= & \frac{\sinh (2 u)+\sin (2 u)-2 \sinh (u)-2 \sin (u)}{2 u(\sin (u) \sinh (2 u)-\sinh (u) \sin (2 u))} \\
\beta_{1,2}= & -\frac{(\sin (u)+\sinh (u))(2 \cos (u) \cosh (u)-\cosh (u)-\cos (u))}{2 u \sinh (u)(\cosh (u)-\cos (u)) \sin (u)} \\
\beta_{2,2}= & \frac{(\cos (u)-1) \sinh (u)+\sin (u)(\cosh (u)-1)}{u(\sin (u) \sinh (2 u)-\sinh (u) \sin (2 u))} \\
\alpha_{1,1}= & -\alpha_{0,1}=\frac{u}{2}(\cot (u)+\operatorname{coth}(u)) \\
\beta_{0,1}= & \frac{b_{01}}{2 u \sinh (u)(-\cosh (u)+\cos (u)) \sin (u)} \\
\beta_{1,1}= & -\frac{b_{11}}{2 u(\sin (u) \sinh (2 u)-\sinh (u) \sin (2 u))} \\
\beta_{2,1}= & \frac{\cosh (2 u) \sin (u)+\cos (2 u) \sinh (u)-\cosh (u) \sin (u)-\sinh (u) \cos (u)}{u(\sin (u) \sinh (2 u)-\sinh (u) \sin (2 u))} \\
& \left.-\frac{11)}{2 u}\right) \\
&
\end{aligned}
$$


with

$$
\begin{aligned}
b_{01}= & \sin (u)-(\sinh (u) \cos (u)+\sin (u)(\cos (u)-1)) \cosh (u) \\
& +\left(-(\cos (u))^{2}+\cos (u)+1\right) \sinh (u)-\sin (u)(\cosh (u))^{2}, \\
b_{11}= & 2 \cosh (2 u) \sin (2 u)-\cosh (2 u) \sin (u)+2 \sinh (2 u) \cos (2 u)-\sin (u) \\
& -\sinh (2 u) \cos (u)-\cos (2 u) \sinh (u)-\sin (2 u) \cosh (u)-\sinh (u), \\
\beta_{0}= & \frac{\cos (u)+\cosh (u)-2}{u^{2}(\cosh (u)-\cos (u))} \\
\beta_{1}= & \frac{(4 \cos (u)-2) \cosh (u)-2 \cos (u)}{u^{2}(-\cosh (u)+\cos (u))} \\
\beta_{2}= & \frac{\cos (u)+\cosh (u)-2}{u^{2}(\cosh (u)-\cos (u))}
\end{aligned}
$$

Remark 2.4. We note that the two coefficients $\beta_{0}$ and $\beta_{2}$ of the main formula of the FFBNM are the same, and thus it is a symmetric formula. The plots of these coefficients are shown in Fig. 1.

For small values of $u$, the coefficients of the FFBNM may be subject to heavy cancellations. In that case the Taylor series expansion of the coefficients must be used (see Lambert [28]). The series expansion of each of the coefficients, up to the twelfth order of approximation, is as follows

$$
\begin{aligned}
& \alpha_{1,3}=-\alpha_{0,3}=1-\frac{u^{4}}{45}-\frac{u^{8}}{4725}-\frac{1382 u^{12}}{638512875} \\
& \beta_{0,3}=-\frac{7}{24}-\frac{73 u^{4}}{40320}-\frac{1717 u^{8}}{95800320}-\frac{85829 u^{12}}{464950886400} \\
& \beta_{1,3}=-\frac{1}{4}-\frac{53 u^{4}}{8640}-\frac{u^{8}}{17920}-\frac{1238269 u^{12}}{2092278988800} \\
& \beta_{2,3}=\frac{1}{24}+\frac{37 u^{4}}{120960}+\frac{331 u^{8}}{95800320}+\frac{145043 u^{12}}{4184557977600} \\
& \alpha_{1,2}=-\alpha_{0,2}=1+\frac{7 u^{4}}{360}+\frac{127 u^{8}}{604800}+\frac{1414477 u^{12}}{653837184000} \\
& \beta_{0,2}=\frac{1}{8}+\frac{211 u^{4}}{120960}+\frac{571 u^{8}}{31933440}+\frac{772349 u^{12}}{4184557977600} \\
& \beta_{1,2}=\frac{5}{12}+\frac{25 u^{4}}{4032}+\frac{535 u^{8}}{9580032}+\frac{1501 u^{12}}{2536095744} \\
& \beta_{2,2}=-\frac{1}{24}-\frac{37 u^{4}}{120960}-\frac{331 u^{8}}{95800320}-\frac{145043 u^{12}}{4184557977600} \\
& \alpha_{1,1}=-\alpha_{0,1}=1-\frac{u^{4}}{45}-\frac{u^{8}}{4725}-\frac{1382 u^{12}}{638512875} \\
& \beta_{0,1}=\frac{1}{24}-\frac{283 u^{4}}{120960}-\frac{227 u^{8}}{13685760}-\frac{71527 u^{12}}{380414361600} \\
& \beta_{1,1}=\frac{13}{12}-\frac{979 u^{4}}{60480}-\frac{1217 u^{8}}{47900160}-\frac{15431 u^{12}}{22992076800} \\
& \beta_{2,1}=\frac{3}{8}-\frac{u^{4}}{4480}+\frac{17 u^{8}}{3548160}+\frac{4841 u^{12}}{154983628800} \\
& =
\end{aligned}
$$




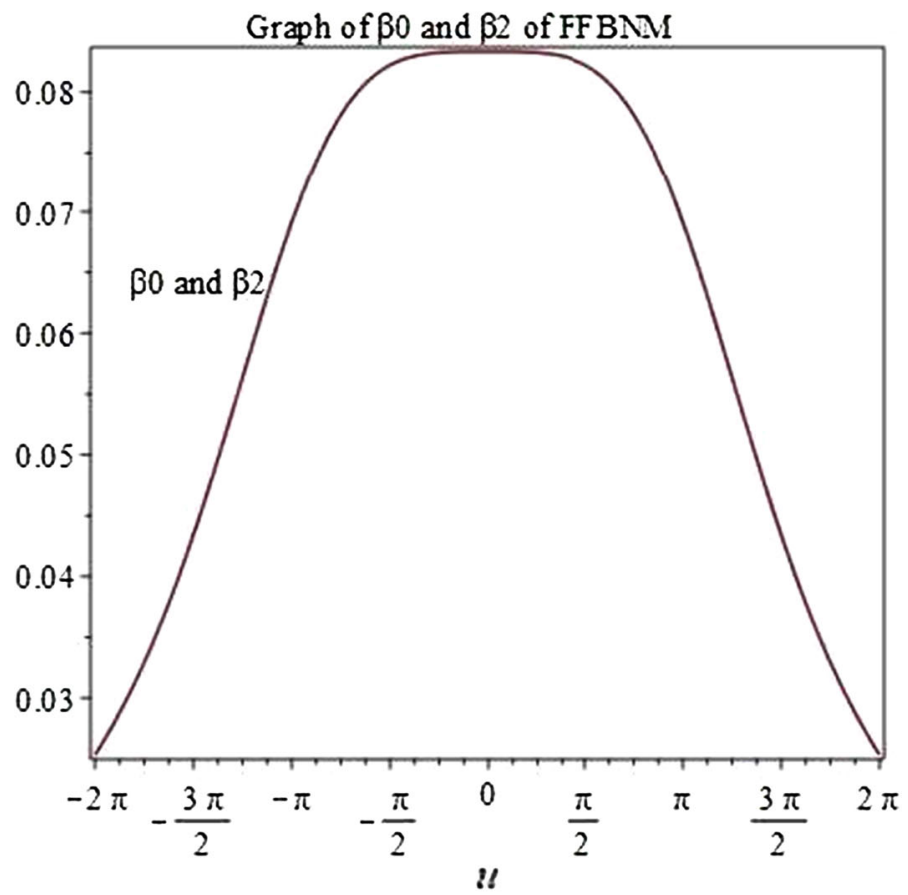

Graph of coefficient $\beta 1$ of FFBNM

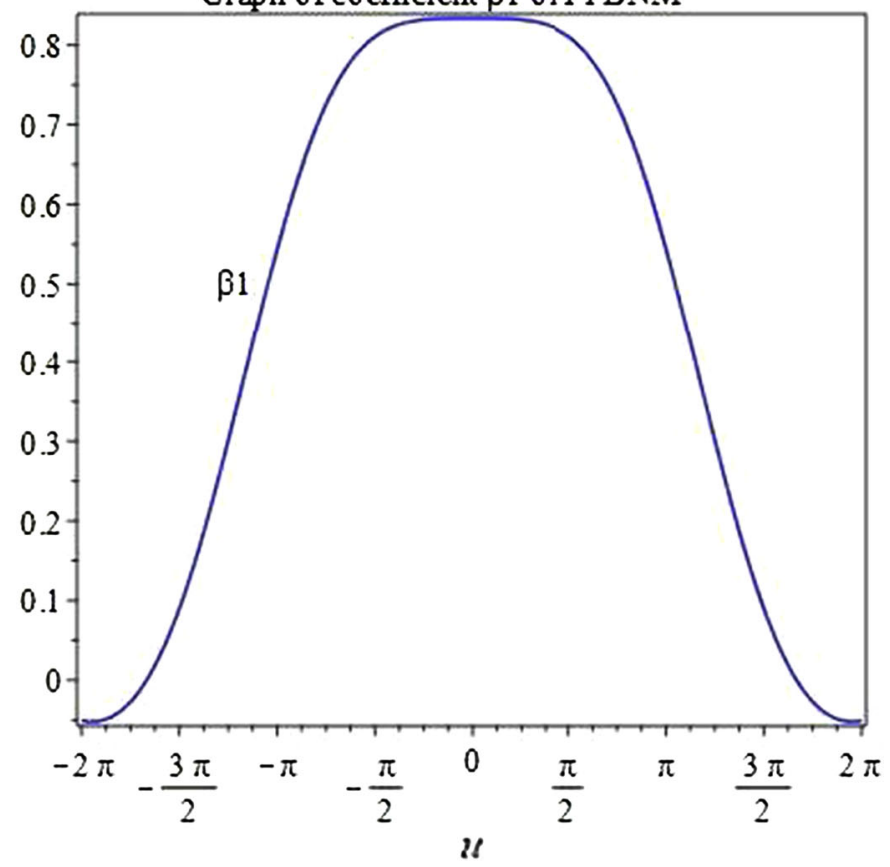

Figure 1. Graphical representation of coefficients $\beta_{i}$ of FFBNM 


$$
\begin{aligned}
& \beta_{0}=\frac{1}{12}-\frac{11 u^{4}}{60480}+\frac{37 u^{8}}{79833600}-\frac{7463 u^{12}}{6276836966400} \\
& \beta_{1}=\frac{5}{6}-\frac{23 u^{4}}{6048}+\frac{17 u^{8}}{1596672}-\frac{17291 u^{12}}{627683696640} \\
& \beta_{2}=\frac{1}{12}-\frac{11 u^{4}}{60480}+\frac{37 u^{8}}{79833600}-\frac{7463 u^{12}}{6276836966400}
\end{aligned}
$$

It is worth to note here that as $u \rightarrow 0$ in the power series expansion of the coefficients (or in the coefficients themselves), the fourth order Numerov method given by

$$
y_{n+2}-2 y_{n+1}+y_{n}=\frac{h^{2}}{12}\left(f_{n}+10 f_{n+1}+f_{n+2}\right)
$$

is recovered from the main method of the FFBNM.

\section{Basic Properties of the FFBNM}

This section discuses the basic properties of the FFBNM which include the Local Truncation Error, Order, Error Constant, Zero-Stability, Convergence, Linear Stability and Region of Stability.

\subsection{Local Truncation Error, Order and Consistency of the FFBNM}

Theorem 3.1. The local truncation error of the main formula in the FFBNM method has the form

$$
L T E=C_{6} h^{6}\left(\omega^{4} y^{\prime \prime}\left(x_{n}\right)-y^{(6)}\left(x_{n}\right)\right)+O\left(h^{7}\right),
$$

where $C_{6}$ is the so called error constant.

Proof. For the main formula, with the assumption that $y(x)$ is a sufficiently differentiable function, we consider the Taylor series expansions of $y\left(x_{n}+j h\right)$, $j=0,1$, and $y^{\prime \prime}\left(x_{n}+j h\right), j=0,1,2$. We replace in the formula the approximate values for the exact ones, that is, $y_{n+j} \rightarrow y\left(x_{n}+j h\right), f_{n+j} \rightarrow$ $y^{\prime \prime}\left(x_{n}+j h\right)$ and substitute the coefficients $\beta_{0}(u), \beta_{1}(u)$ and $\beta_{2}(u)$ given in (2.13) into the last formula in (2.9). After simplifying, we obtain

$$
\begin{aligned}
L T E & =y\left(x_{n}+2 h\right)-\left(y\left(x_{n}\right)-2 y\left(x_{n}+h\right)+h^{2} \sum_{j=0}^{2} \beta_{j}(u) y^{\prime \prime}\left(x_{n}+j h\right)\right) \\
& =C_{6} h^{6}\left(\omega^{4} y^{\prime \prime}\left(x_{n}\right)-y^{(6)}\left(x_{n}\right)\right)+O\left(h^{7}\right) .
\end{aligned}
$$

Following this procedure, the local truncation error is obtained for each of the formulas in (2.9). These errors are given by

$$
L T E=\left\{\begin{array}{l}
\frac{-h^{5}}{45}\left(y^{(5)}\left(x_{n}\right)-\omega^{4} y^{\prime}\left(x_{n}\right)\right)+O\left(h^{6}\right) \\
\frac{7 h^{5}}{360}\left(y^{(5)}\left(x_{n}\right)-\omega^{4} y^{\prime}\left(x_{n}\right)\right)+O\left(h^{6}\right) \\
\frac{-h^{5}}{45}\left(y^{(5)}\left(x_{n}\right)-\omega^{4} y^{\prime}\left(x_{n}\right)\right)+O\left(h^{6}\right) \\
\frac{-h^{6}}{240}\left(y^{(6)}\left(x_{n}\right)-\omega^{4} y^{\prime \prime}\left(x_{n}\right)\right)+O\left(h^{7}\right)
\end{array}\right.
$$


which indicates that the order of the main formula is $p=4$ and the order of each complimentary formula is $p=3$. The order of convergence of the FFBNM will be analyzed below.

Theorem 3.2. The local truncation error of the main formula of the FFBNM preserves its basis functions, that is, when the solution of the problem in (1.1) is a linear combination of the basis functions $\left\{\sigma_{i}\right\}_{i=0}^{4}$ the local truncation error vanishes.

Proof. Solving the differential equation $y^{(6)}(x)-\omega^{4} y^{\prime \prime}(x)=0$ results in the following fundamental set of solutions $\{1, x, \sin (\omega x), \cos (\omega x), \sinh (\omega x)$, $\cosh (\omega x)\}$, which contains the basis functions of the FFBNM, from which the statement follows immediately.

Remark 3.3. Following the definition by Lambert [28], a numerical approach for solving (1.1) is said to be consistent if it has an order greater than one. We thus have that the FFBNM is consistent.

\subsection{Analysis of Convergence of the FFBNM}

The analysis of convergence of the FFBNM is done following the guidelines by Jain and Aziz [20], Jator and Li [24] and Abdulganiy et al. [1].

Theorem 3.4. Let $\overline{\mathrm{Y}}$ be a vector that approximates the true solution vector $Y$, where $\overline{\mathrm{Y}}$ is the solution of the system obtained from the FFBNM given by the equations in (2.9) on the successive block intervals $\left[x_{0}, x_{2}\right]$, $\left[x_{2}, x_{4}\right], \ldots,\left[x_{N-2}, x_{N}\right]$, with $N$ even.

If we denote by $E=\left(e_{1}, \ldots, e_{N}, h e_{1}^{\prime}, \ldots, h e_{N}^{\prime}\right)^{T}$ the error vector, where $e_{j}=y\left(x_{j}\right)-y_{j}$ and $h e_{j}^{\prime}=h y^{\prime}\left(x_{j}\right)-h y_{j}^{\prime}, j=1,2, \ldots, N$, assuming that the exact solution is sufficiently differentiable on $\left[x_{0}, x_{N}\right]$, then, for sufficiently small $h$ the FFBNM is a third-order convergent method, that is,

$$
\|\mathrm{E}\|=\|\overline{\mathrm{Y}}-\mathrm{Y}\|=O\left(h^{3}\right) .
$$

Proof. Let the $(2 N \times N)$-matrices of coefficients obtained from the FFBNM method be defined as follows:

$$
P_{1}=\left(\begin{array}{lllllllllll}
-\alpha_{1,3} & 0 & 0 & 0 & 0 & 0 & 0 & \cdots & 0 & 0 & 0 \\
-\alpha_{1,2} & 0 & 0 & 0 & 0 & 0 & 0 & \cdots & 0 & 0 & 0 \\
-\alpha_{1,1} & 0 & 0 & 0 & 0 & 0 & 0 & \cdots & 0 & 0 & 0 \\
-2 & 1 & 0 & 0 & 0 & 0 & 0 & \cdots & 0 & 0 & 0 \\
0 & -\alpha_{0,3} & -\alpha_{1,3} & 0 & 0 & 0 & 0 & \cdots & 0 & 0 & 0 \\
0 & -\alpha_{0,2} & -\alpha_{1,2} & 0 & 0 & 0 & 0 & \cdots & 0 & 0 & 0 \\
0 & -\alpha_{0,1} & -\alpha_{1,1} & 0 & 0 & 0 & 0 & \cdots & 0 & 0 & 0 \\
0 & 1 & -2 & 1 & 0 & 0 & 0 & \cdots & 0 & 0 & 0 \\
\vdots & \vdots & \vdots & \vdots & \vdots & \vdots & \vdots & \ddots & \vdots & \vdots & \vdots \\
0 & 0 & 0 & 0 & 0 & 0 & 0 & \cdots & -\alpha_{0,3} & -\alpha_{1,3} & 0 \\
0 & 0 & 0 & 0 & 0 & 0 & 0 & \cdots & -\alpha_{0,2} & -\alpha_{1,2} & 0 \\
0 & 0 & 0 & 0 & 0 & 0 & 0 & \cdots & -\alpha_{0,1} & -\alpha_{1,1} & 0 \\
0 & 0 & 0 & 0 & 0 & 0 & 0 & \cdots & 1 & -2 & 1
\end{array}\right)
$$




$$
\begin{aligned}
& P_{2}=\left(\begin{array}{ccccccccccc}
0 & 0 & 0 & 0 & 0 & 0 & 0 & \cdots & 0 & 0 & 0 \\
1 & 0 & 0 & 0 & 0 & 0 & 0 & \cdots & 0 & 0 & 0 \\
0 & 1 & 0 & 0 & 0 & 0 & 0 & \cdots & 0 & 0 & 0 \\
0 & 0 & 0 & 0 & 0 & 0 & 0 & \cdots & 0 & 0 & 0 \\
0 & 1 & 0 & 0 & 0 & 0 & 0 & \cdots & 0 & 0 & 0 \\
0 & 0 & 1 & 0 & 0 & 0 & 0 & \cdots & 0 & 0 & 0 \\
0 & 0 & 0 & 1 & 0 & 0 & 0 & \cdots & 0 & 0 & 0 \\
0 & 0 & 0 & 0 & 0 & 0 & 0 & \cdots & 0 & 0 & 0 \\
\vdots & \vdots & \vdots & \vdots & \vdots & \vdots & \vdots & \ddots & \vdots & \vdots & \vdots \\
0 & 0 & 0 & 0 & 0 & 0 & 0 & \cdots & 1 & 0 & 0 \\
0 & 0 & 0 & 0 & 0 & 0 & 0 & \cdots & 0 & 1 & 0 \\
0 & 0 & 0 & 0 & 0 & 0 & 0 & \cdots & 0 & 0 & 1 \\
0 & 0 & 0 & 0 & 0 & 0 & 0 & \cdots & 0 & 0 & 0
\end{array}\right), \\
& Q=h^{2}\left(\begin{array}{lllllllllll}
\beta_{1,3} & \beta_{2,3} & 0 & 0 & 0 & 0 & 0 & \cdots & 0 & 0 & 0 \\
\beta_{1,2} & \beta_{2,2} & 0 & 0 & 0 & 0 & 0 & \cdots & 0 & 0 & 0 \\
\beta_{1,1} & \beta_{1,2} & 0 & 0 & 0 & 0 & 0 & \cdots & 0 & 0 & 0 \\
\beta_{1} & \beta_{2} & 0 & 0 & 0 & 0 & 0 & \cdots & 0 & 0 & 0 \\
0 & \beta_{0,3} & \beta_{1,3} & \beta_{2,3} & 0 & 0 & 0 & \cdots & 0 & 0 & 0 \\
0 & \beta_{0,2} & \beta_{1,2} & \beta_{2,2} & 0 & 0 & 0 & \cdots & 0 & 0 & 0 \\
0 & \beta_{0,1} & \beta_{1,1} & \beta_{2,1} & 0 & 0 & 0 & \cdots & 0 & 0 & 0 \\
0 & \beta_{0} & \beta_{1} & \beta_{2} & 0 & 0 & 0 & \cdots & 0 & 0 & 0 \\
\vdots & \vdots & \vdots & \vdots & \vdots & \vdots & \vdots & \ddots & \vdots & \vdots & \vdots \\
0 & 0 & 0 & 0 & 0 & 0 & 0 & \cdots & \beta_{0,3} & \beta_{1,3} & \beta_{2,3} \\
0 & 0 & 0 & 0 & 0 & 0 & 0 & \cdots & \beta_{0,2} & \beta_{1,2} & \beta_{2,2} \\
0 & 0 & 0 & 0 & 0 & 0 & 0 & \cdots & \beta_{0,1} & \beta_{1,1} & \beta_{2,1} \\
0 & 0 & 0 & 0 & 0 & 0 & 0 & \cdots & \beta_{0} & \beta_{1} & \beta_{2}
\end{array}\right),
\end{aligned}
$$

and the $2 N$-vector containing the known values given by

$$
\begin{aligned}
C= & \left(-\alpha_{0,3} y_{0}+h y_{0}^{\prime}-h^{2} \beta_{0,3} f_{0},-\alpha_{0,2} y_{0}-h^{2} \beta_{0,2} f_{0},\right. \\
& \left.-\alpha_{0,1} y_{0}-h^{2} \beta_{0,1} f_{0}, y_{0}-h^{2} \beta_{0} f_{0}, 0, \ldots, 0\right)^{T} .
\end{aligned}
$$

Let consider the vectors of exact values

$$
\begin{aligned}
& Y=\left(y\left(x_{1}\right), \ldots, y\left(x_{N}\right), h y^{\prime}\left(x_{1}\right), \ldots, h y^{\prime}\left(x_{N}\right)\right), \\
& F=\left(f\left(x_{1}, y\left(x_{1}\right), y^{\prime}\left(x_{1}\right)\right), \ldots, f\left(x_{N}, y\left(x_{N}\right), y^{\prime}\left(x_{N}\right)\right)\right),
\end{aligned}
$$

the vectors of approximate values

$$
\begin{aligned}
\bar{Y} & =\left(y_{1}, \ldots, y_{N}, h y_{1}^{\prime}, \ldots, h y_{N}^{\prime}\right), \\
\bar{F} & =\left(f_{1}, \ldots, f_{N}\right),
\end{aligned}
$$

and the vector of local truncation errors $L(h)=\left(L_{1}, \ldots, L_{2 N}\right)$.

Remark 3.5. Note that to form the global method the formulas in (2.9) are considered for $n=0,2,4, \ldots, N-2$, so we have a total of $2 N$ formulas and $2 N$ unknowns. Thus, we have a total of $2 N$ truncation errors provided by the formulas in (3.1), which form the vector $L(h)$.

Taking the $(2 N \times 2 N)$-matrix $P=\left(P_{1} \mid P_{2}\right)$, the exact form of the system formed by the formulas in (2.9) along the two-step block intervals on $\left[x_{0}, x_{N}\right]$ 
is

$$
P Y-Q F+C=L(h) .
$$

On the other hand, the system may be written as

$$
P \overline{\mathrm{Y}}-Q \bar{F}+C=0 \text {. }
$$

Subtracting equation (3.3) from equation (3.2) we obtain

$$
P(Y-\overline{\mathrm{Y}})-Q(F-\bar{F})=L(h),
$$

and having in mind that $E=\left(e_{1}, \ldots, e_{N}, h e_{1}^{\prime}, \ldots, h e_{N}^{\prime}\right)^{T}$, the above equation becomes

$$
P E-Q(F-\bar{F})=L(h) .
$$

Applying the Mean-Value Theorem, we obtain that $F-\bar{F}=J E$, where $J$ is the $(N \times 2 N)$-matrix given as

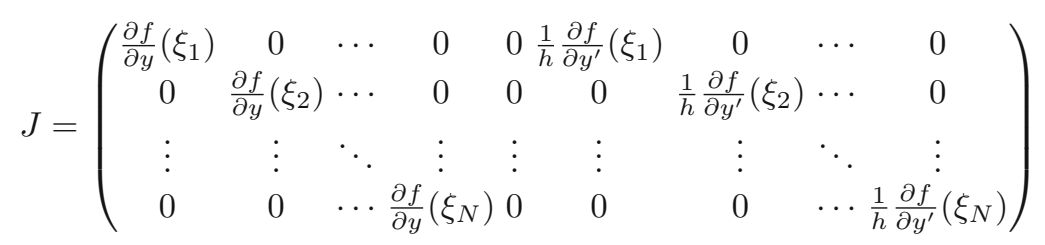

and the partial derivatives are applied at intermediate points $\left\{\xi_{i}\right\}_{i=1}^{N}$, which are on each corresponding line joining $\left(x_{i}, y\left(x_{i}\right), y^{\prime}\left(x_{i}\right)\right)$ to $\left(x_{i}, y_{i}, y_{i}^{\prime}\right)$. In view of this, the equation in (3.5) can be written as

$$
(P-Q J) E=L(h) .
$$

Let $M$ denote the matrix $M=-Q J$. Then, we have that

$$
(P+M) E=L(h) .
$$

For sufficiently small $h$, the matrix $P+M$ is invertible (see [20,24]). Therefore, if we denote by

$$
(P+M)^{-1}=D
$$

and consider the maximum norm, we can obtain after expanding in Taylor series the terms in $D$ that $\|D\|=O\left(h^{-2}\right)$. Finally, we have that

$$
\begin{aligned}
\|E\| & =\|D L(h)\|=\|D\|\|L(h)\| \\
& =\left|O\left(h^{-2}\right)\right|\left|O\left(h^{5}\right)\right|=O\left(h^{3}\right) .
\end{aligned}
$$

Therefore, the FFBNM is a third-order convergent method.

Remark 3.6. In case of a special second-order equation, that is, $y^{\prime \prime}=f(x, y)$, where the first derivative is absent, we can proceed similarly. Nevertheless, in view of the form of the vector $L(h)$ we see that, assuming sufficient smoothness, we obtain a higher order of convergence:

$$
\left|e_{j}\right|=\left|y\left(x_{j}\right)-y_{j}\right|=\left|\mathcal{O}\left(h^{-2}\right)\right|\left|\mathcal{O}\left(h^{6}\right)\right|=O\left(h^{4}\right), \quad j=1,2 \ldots N .
$$




\subsection{Stability of the FFBNM}

Following Fatunla [13], the FFBNM can be represented in the following block matrix form

$$
\begin{aligned}
\left(A^{(1)} \otimes I\right) Y_{\mu+1}= & \left(A^{(0)} \otimes I\right) \mathrm{Y}_{\mu}+h^{2}\left(B^{(1)} \otimes I\right) F_{\mu+1} \\
& +h^{2}\left(B^{(0)} \otimes I\right) F_{\mu}
\end{aligned}
$$

where $Y_{\mu+1}=\left(y_{n+1}, y_{n+2}, h y_{n+1}^{\prime}, h y_{n+2}^{\prime}\right)^{T}, Y_{\mu}=\left(y_{n-1}, y_{n} h y_{n-1}^{\prime}, h y_{n}^{\prime}\right)^{T}$, $F_{\mu+1}=\left(f_{n+1}, f_{n+2}, h f_{n+1}^{\prime}, h f_{n+2}^{\prime}\right)^{T}, F_{\mu}=\left(f_{n-1}, f_{n}, h f_{n-1}^{\prime}, h f_{n}^{\prime}\right)^{T}, I$ is the identity matrix of dimension four, $\otimes$ denotes the Kronecker product of matrices, and $A^{(0)}, A^{(1)}, B^{(0)}, B^{(1)}$ are $4 \times 4$ matrices obtained from the coefficients of the method, and given by

$$
\begin{aligned}
A^{(1)} & =\left(\begin{array}{cccc}
-\alpha_{1,3} & 0 & 0 & 0 \\
-\alpha_{1,2} & 0 & 1 & 0 \\
-\alpha_{1,1} & 0 & 0 & 1 \\
-2 & 1 & 0 & 0
\end{array}\right), A^{(0)}=\left(\begin{array}{cccc}
0 & \alpha_{0,3} & 0 & -1 \\
0 & \alpha_{0,2} & 0 & 0 \\
0 & \alpha_{0,1} & 0 & 0 \\
0 & -1 & 0 & 0
\end{array}\right), \\
B^{(1)} & =\left(\begin{array}{cccc}
\beta_{1,3} & \beta_{2,3} & 0 & 0 \\
\beta_{1,2} & \beta_{2,2} & 0 & 0 \\
\beta_{1,1} & \beta_{2,1} & 0 & 0 \\
\beta_{1} & \beta_{2} & 0 & 0
\end{array}\right), B^{(0)}=\left(\begin{array}{cccc}
0 & \beta_{0,3} & 0 & 0 \\
0 & \beta_{0,2} & 0 & 0 \\
0 & \beta_{0,1} & 0 & 0 \\
0 & \beta_{0} & 0 & 0
\end{array}\right) .
\end{aligned}
$$

\subsubsection{Zero Stability of the FFBNM.}

Definition 3.7. A block method is zero-stable if the roots of its first characteristic polynomial, $\rho(R)=\operatorname{det}\left(R A^{(1)}-A^{(0)}\right)$, are of modulus less than or equal to one, and for those which modulus one the multiplicity is not greater than two (see Fatunla [12]).

Proposition 3.8. The FFBNM is zero-stable.

Proof. From the normalized first characteristic polynomial of the FFBNM, we have that

$$
R A^{(1)}-A^{(0)}=\left[\begin{array}{cccc}
-R \alpha_{1,3} & -\alpha_{0,3} & 0 & 1 \\
-R \alpha_{1,2} & -\alpha_{0,2} & R & 0 \\
-R \alpha_{1,1} & -\alpha_{0,1} & 0 & R \\
-2 R & R+1 & 0 & 0
\end{array}\right]
$$

so that the characteristic equation is $\rho(R)=\operatorname{det}\left(R A^{(1)}-A^{(0)}\right)=0$, that is, $-\eta R^{2}(R-1)^{2}=0$, with $\eta=\alpha_{0,1}$. Consequently, according to the above definition the method is zero-stable.

3.3.2. Linear Stability of the FFBNM. Applying the FFBNM specified by the formulas in (2.9) to the test equation $y^{\prime \prime}=\lambda^{2} y$ and letting $z=\lambda h$ yields

$$
Y_{\mu+1}=M(z, u) Y_{\mu}
$$

where

$$
M(z, u)=\left(A^{(1)}-z B^{(1)}\right)^{-1}\left(A^{(0)}+z B^{(0)}\right)
$$




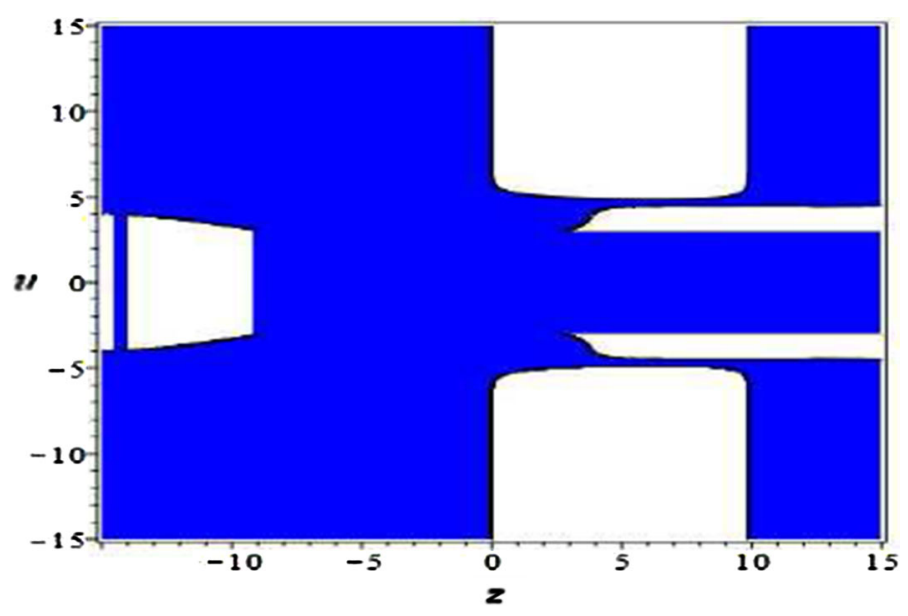

Figure 2. $z-u$ stability region for the FFBNM

is the so-called stability matrix or amplification matrix, which determines the stability of the FFBNM. The amplification matrix $M(z, u)$ for FFBNM has eigenvalues given by $\left(\varphi_{1}, \varphi_{2}, \varphi_{3}, \varphi_{4}\right)=\left(0,0,0, \varphi_{4}\right)$, where $\varphi_{4}(z, u)=\frac{p_{4}(z, u)}{q_{4}(z, u)}$ is the stability function.

Definition 3.9. The region of linear stability of the method is the domain in the $z-u$ plane in which the spectral radius of the amplification matrix, $\rho(M(z, u))$, verifies $|\rho(M(z, u))| \leq 1$ (see Jator [27]).

The $z-u$ stability region constructed for the FFBNM is plotted in Fig. 2.

\section{Implementation of the FFBNM}

The FFBNM is implemented in a block-by-block fashion for solving the problem in (1.1) on $\left[x_{0}, x_{N}\right]$. We use the formulas in (2.9) to obtain the approximations $y_{n+1}, y_{n+2}$, and $y_{n+1}^{\prime}, y_{n+2}^{\prime}$, simultaneously over the sequence of non overlapping intervals $\left[x_{0}, x_{2}\right],\left[x_{2}, x_{4}\right], \ldots,\left[x_{N-2}, x_{N}\right]$, with $N$ even. When the method is applied on $\left[x_{0}, x_{2}\right]$ the approximate values $\left\{y_{1}, y_{2}, y^{\prime}{ }_{1}, y^{\prime}{ }_{2}\right\}$ are obtained simultaneously, assuming that $y_{0}$ and $y_{0}^{\prime}$ are known from the IVP in $(1.1)$; on the next interval $\left[x_{2}, x_{4}\right]$ we obtain the values $\left\{y_{3}, y_{4}, y^{\prime}{ }_{3}, y^{\prime}{ }_{4}\right\}$ simultaneously, since $y_{2}$ and $y_{2}^{\prime}$ are known from the previous block. This process continues until we obtain the numerical solution of equation (1) on the entire interval of integration. We emphasizes that this procedure makes the FFBNM a self-starting method which does not suffer the disadvantage of predictor-corrector modes. We also note that the computations were carried out using a written code in Maple 2016.2 on a Laptop with

1. 64 bit Windows 10 Pro Operating System,

2. Intel (R) Celeron CPU N3060 @ 1.60GHz processor, and

3. 4.00GB RAM memory. 
Table 1. Comparison of results for example 1

\begin{tabular}{|c|c|c|c|c|c|c|c|c|c|}
\hline \multicolumn{2}{|c|}{ FFBNM } & \multicolumn{2}{|l|}{ EM8 } & \multicolumn{2}{|c|}{ HLMM } & \multicolumn{2}{|c|}{ BTDF8 } & \multicolumn{2}{|c|}{ BTDF10 } \\
\hline NFE & $\mathrm{CD}$ & NFE & $\mathrm{CD}$ & NFE & CD & NFE & CD & $\mathrm{NFE}$ & CD \\
\hline 126 & 9.1 & 2000 & 5.7 & 301 & 7.1 & 212 & 5.5 & 282 & 7.2 \\
\hline 251 & 11.2 & 3000 & 8.2 & 601 & 9.5 & 422 & 8.3 & 562 & 11.0 \\
\hline 501 & 12.7 & 4000 & 9.6 & 1201 & 11.5 & 842 & 10.9 & 1122 & 11.8 \\
\hline 751 & 13.9 & 5000 & 10.5 & 1801 & 11.8 & 1262 & 11.8 & 1682 & 11.9 \\
\hline 1001 & 14.1 & 6000 & 10.9 & 2401 & 11.8 & 1682 & 11.8 & 2242 & 11.9 \\
\hline 1251 & 15.2 & 7000 & 11.1 & 3601 & 11.9 & 2522 & 11.9 & 3362 & 11.9 \\
\hline
\end{tabular}

For non-linear problems, the code was enhanced by the feature fsolve in Maple 2016.2.

\subsection{Numerical Examples}

This subsection examines the effectiveness of the FFBNM. Seven well-known numerical examples with oscillating solutions that have appeared in the literature are considered. In the numerical investigations, we used two criteria: accuracy and efficiency. A measure of accuracy is investigated using the maximum error of the approximate solution defined as ErrMax $=$ $\max \left\|y\left(x_{n}\right)-y_{n}\right\|_{\infty}$, where $y\left(x_{n}\right)$ is the exact solution and $y_{n}$ is the numerical solution obtained using the FFBNM. The computational efficiency criterion is specified at each of the considered examples.

\subsection{Example 1}

Consider the nonlinear Duffing equation given by

$$
y^{\prime \prime}+y+y^{3}=B \cos (\Omega x)
$$

whose exact solution is unknown. Its approximate theoretical solution obtained by Van Dooren [44] is given by $y(x)=C_{1} \cos (\Omega x)+C_{2} \cos (3 \Omega x)+$ $C_{3} \cos (5 \Omega x)+C_{4} \cos (7 \Omega x)$ and the suitable initial conditions are $y(0)=$ $C_{0}, y^{\prime}(0)=0$, where $\Omega=1.01, B=0.002, C_{0}=0.200426728069, C_{1}=$ $0.200179477536, C_{2}=0.246946143 \times 10^{-3}, C_{3}=0.304016 \times 10^{-6}, C_{4}=0.374 \times$ $10^{-9}$. This problem has been considered by Tsitouras [41], Jator [21] and Jator et al. [27] in the interval $\left[0, \frac{20.5 \pi}{1.01}\right]$, with $\omega=1.01$ using an explicit eighth order method (EM8), a seventh order hybrid method (HLMM), and two third derivative block methods of orders eighth and tenth (BTDF8 and BTDF10), respectively. Table 1 shows the maximum norm of the global error for the $y$-component of FFBNM given in the form $10^{-C D}$, where CD denotes the number of decimal digits in comparison with the aforementioned methods.

The accuracy of FFBNM together with its efficiency, measured by $\log _{10}(\operatorname{ErrMax})$, against the logarithm of the number of function evaluations, $\log _{10}(N F E)$, are presented be means of the efficiency curves in Fig. 3 . 
Table 2. Comparison of Results for Example 2

\begin{tabular}{|c|c|c|c|c|c|c|c|c|c|c|c|}
\hline \multicolumn{3}{|c|}{ FFBNM } & \multicolumn{3}{|c|}{ TRI5 } & \multicolumn{3}{|c|}{ DIS6 } & \multicolumn{3}{|c|}{ TFARKN } \\
\hline $\mathrm{N}$ & CD & NFE & $\mathrm{N}$ & $\mathrm{CD}$ & NFE & $\mathrm{N}$ & CD & NFE & $\mathrm{N}(\mathrm{rej})$ & CD & $\mathrm{NFE}$ \\
\hline 40 & 6.9 & 41 & 40 & 4.7 & 230 & 40 & 3.2 & 300 & $29(6)$ & 2.8 & 250 \\
\hline 80 & 7.1 & 81 & 80 & 6.9 & 300 & 80 & 5.3 & 450 & $88(9)$ & 5.3 & 450 \\
\hline 160 & 8.8 & 161 & 160 & 8.1 & 500 & 160 & 7.6 & 700 & $262(8)$ & 7.9 & 1000 \\
\hline 320 & 10.6 & 321 & 320 & 10.0 & 950 & 320 & 9.5 & 1250 & $811(4)$ & 10.4 & 3250 \\
\hline
\end{tabular}

As is evident from the results in Table 1 and the efficiency curves in Figure 3, the FFBNM is a more efficient integrator for the considered nonlinear Duffing equation than the other methods used for comparison, even for the higher order method BTDF10 in Jator et al. [27].

\subsection{Example 2}

Consider the nonlinear perturbed system on the range $[0,10]$ with $\epsilon=10^{-3}$

$$
\begin{aligned}
y_{1}^{\prime \prime} & =\epsilon \varphi_{1}(x)-25 y_{1}-\epsilon\left(y_{1}^{2}+y_{2}^{2}\right) \quad y_{1}(0)=1, y_{1}^{\prime}(0)=0, y_{2}^{\prime \prime} \\
& =\epsilon \varphi_{2}(x)-25 y_{2}-\epsilon\left(y_{1}^{2}+y_{2}^{2}\right) \quad y_{2}(0)=\epsilon, y_{2}^{\prime}(0)=5,
\end{aligned}
$$

where

$$
\begin{aligned}
& \varphi_{1}(x)=1+\epsilon^{2}+2 \epsilon \sin \left(5 x+x^{2}\right)+2 \cos \left(x^{2}\right)+\left(25-4 x^{2}\right) \sin \left(x^{2}\right), \\
& \varphi_{2}(x)=1+\epsilon^{2}+2 \epsilon \sin \left(5 x+x^{2}\right)-2 \sin \left(x^{2}\right)+\left(25-4 x^{2}\right) \cos \left(x^{2}\right) .
\end{aligned}
$$

The solution in closed form is given by $y_{1}(x)=\cos (5 x)+\epsilon \sin \left(x^{2}\right), y_{2}(x)=$ $\sin (5 x)+\epsilon \cos \left(x^{2}\right)$, which represents a periodic motion of constant frequency with a small perturbation of variable frequency. This problem was selected to show the performance of the FFBNM on a nonlinear perturbed system. Thus, we choose $\omega=5$ as the fitting frequency. The errors of the FFBNM were compared with a fifth order Trigonometrically-Fitted Runge-Kutta-Nyström (TFARKN) method by Fang et al. [9], a fifth order trigonometrically-fitted explicit method (TRI5) by Fang and Wu [8], and a sixth order hybrid method with dissipation order seven (DIS6) in [17], as presented in Table 2.

Details of the results given in Table 2 and the efficiency curves plotting the $\log _{10}$ (ErrMax) versus the $\log _{10}$ (NFE) in Fig. 4, reveal that the FFBNM is an efficient integrator for the nonlinear perturbed system.

\subsection{Example 3}

Consider the periodically forced nonlinear IVP

$$
\left\{\begin{array}{c}
y^{\prime \prime}+y^{3}+y=(\cos (t)+\epsilon \sin (10 t))^{3}-99 \epsilon \sin (10 t), 0 \leq t \leq 1000 \\
y(0)=1, y^{\prime}(0)=10 \epsilon
\end{array}\right.
$$

with $\epsilon=10^{-10}$ and whose analytic solution $y(t)=\cos (t)+\epsilon \sin (10 t)$ describes a periodic motion of low frequency with a small perturbation of high frequency. For this problem, $\omega=1$ is selected. The results of FFBNM in comparison to the TFARKN by Fang et al. [9], the EFRK by Franco [14] 

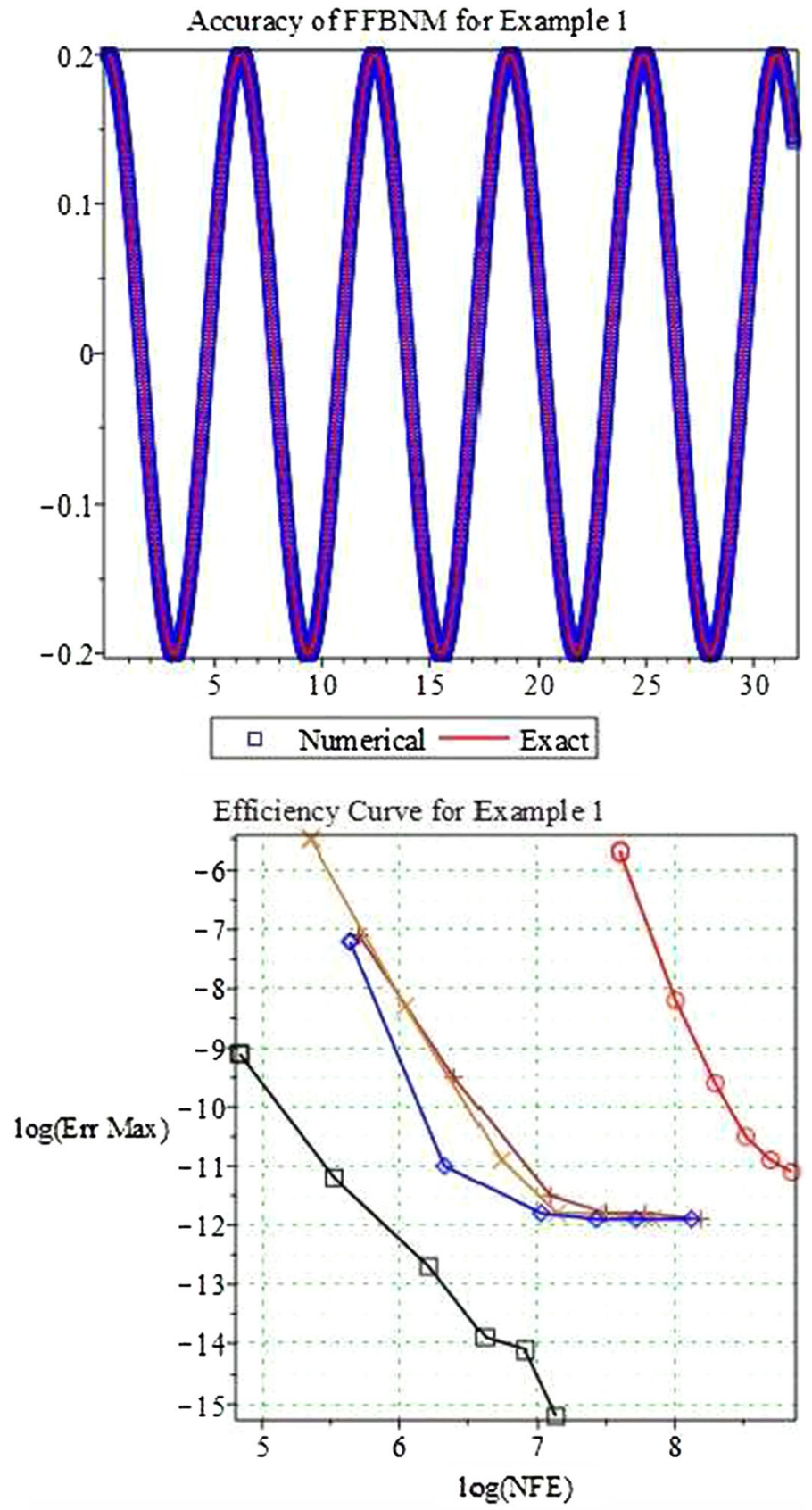

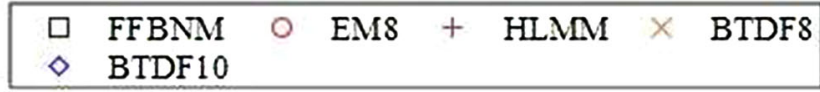

Figure 3. The graphical representation of solution to Example 1 and efficiency curves 
Accuracy ofFFBNM for Example 2

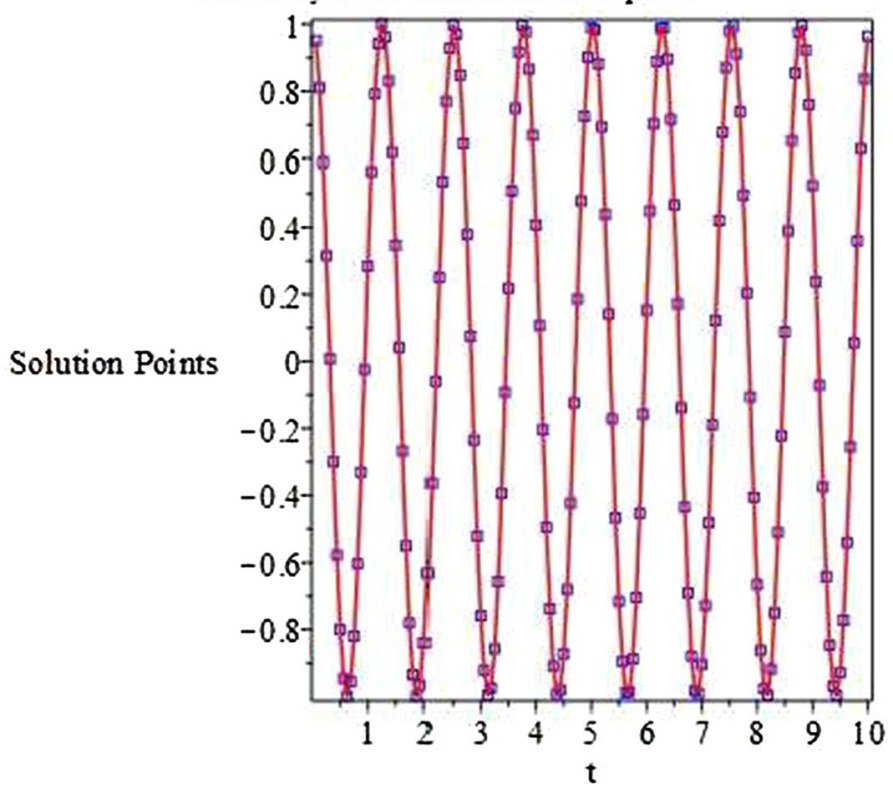

- Numerical y1 - Exact y1

Efficiency Curve for Example 2

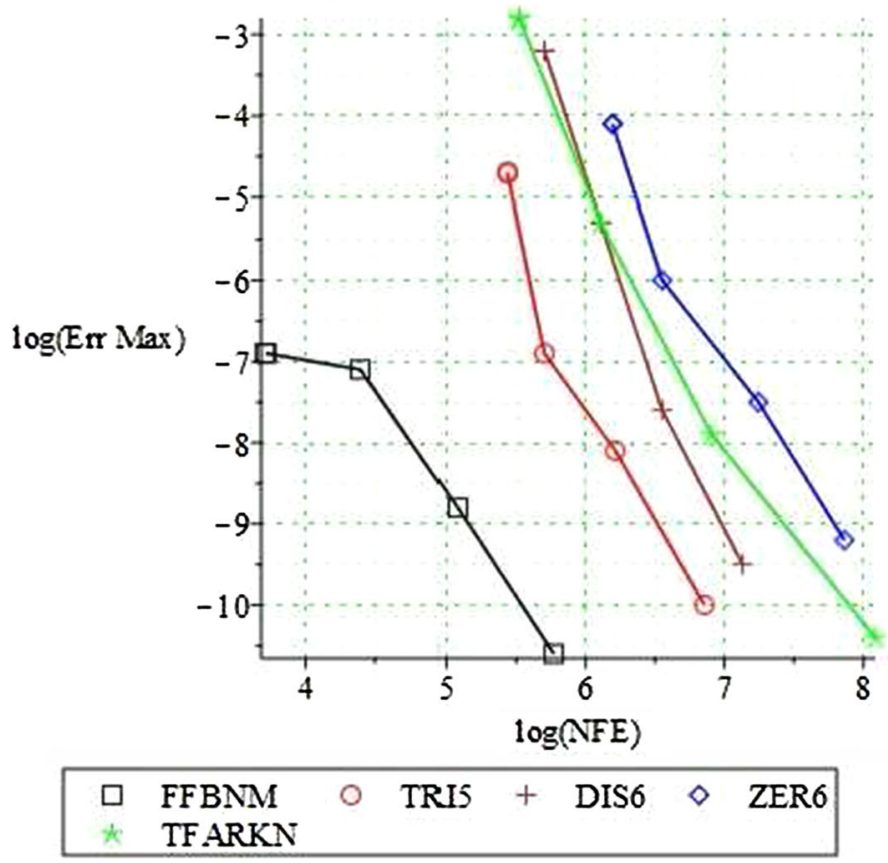

Figure 4. The graphical representation of solution to Example 2, and efficiency curves 
Accuracy of FFBNM for Example 3
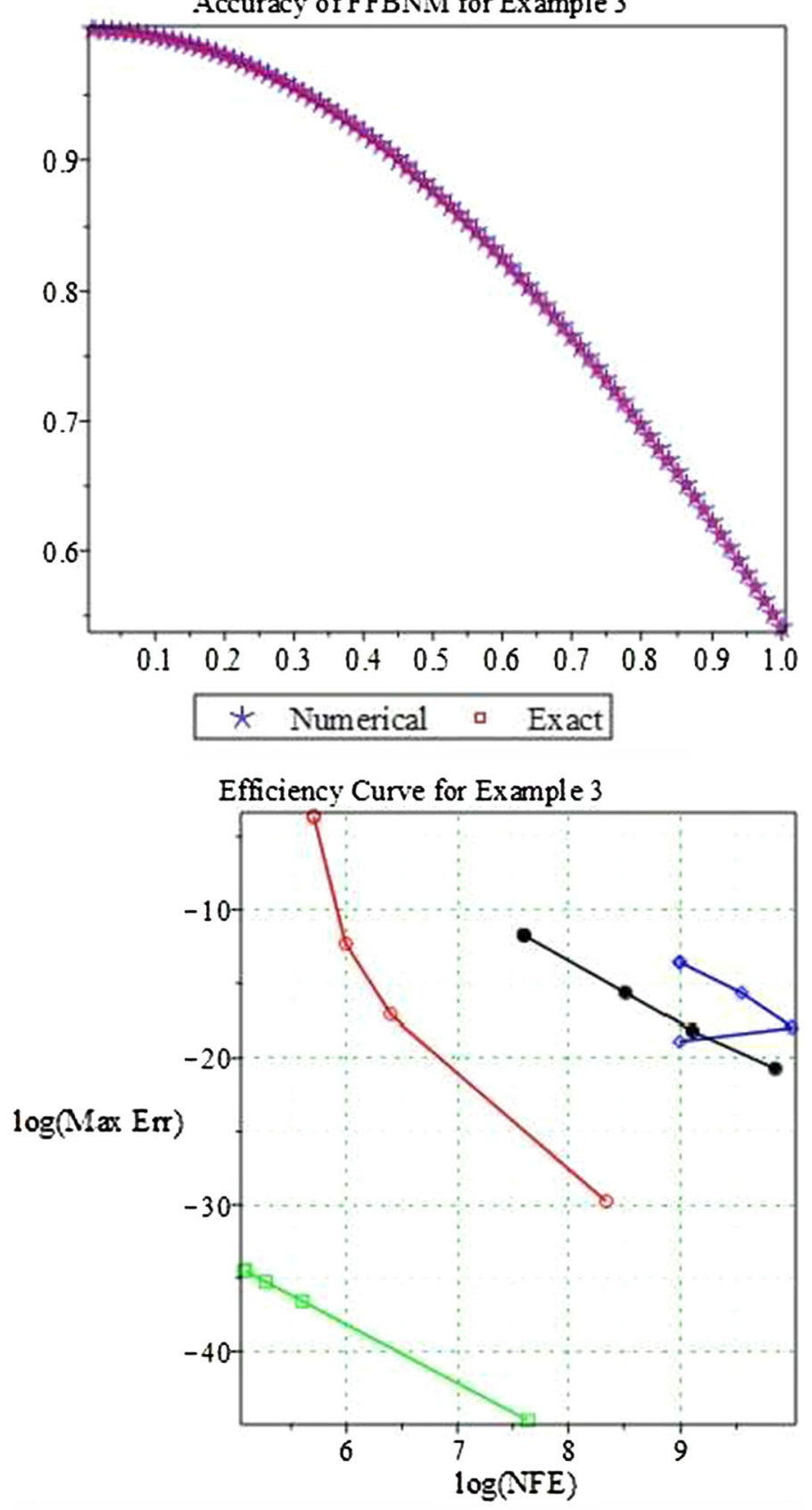

- FFBNM $\odot$ TFARKN $\diamond$ EFRK $~ E F R K N$

Figure 5. The graphical representation of solution to Example 3 , and efficiency curves 
Table 3. Comparison of numerical results for example 3

\begin{tabular}{|c|c|c|c|c|c|c|c|}
\hline FFBNM & & TFARKN & & EFRK & & EFRKN & \\
\hline Error & $\mathrm{NFE}$ & Error & NFE & Error & NFE & Error & NFE \\
\hline $.04 \times 10^{-15}$ & & $10^{-2}$ & 30 & 1.26 & 81 & $10^{-6}$ & \\
\hline 84 & 195 & 4.47 & 400 & 1. & 14000 & $10^{-7}$ & 50 \\
\hline $1.29 \times 10^{-16}$ & 271 & $3.72 \times 10^{-8}$ & 600 & $1.58 \times 10^{-8}$ & 22000 & $1.26 \times 10^{-8}$ & 9000 \\
\hline $3.74 \times 10^{-20}$ & 2071 & $1.17 \times 10^{-13}$ & 4200 & $6.31 \times 10^{-9}$ & 38000 & $1.00 \times 10^{-9}$ & 19000 \\
\hline
\end{tabular}

Table 4. Comparison of numerical results for example 4

\begin{tabular}{|c|c|c|c|c|c|c|c|c|c|}
\hline \multicolumn{2}{|c|}{ FFBNM } & \multicolumn{2}{|c|}{ ETFFSH6S } & \multicolumn{2}{|c|}{ ARK4 } & \multicolumn{2}{|c|}{ ARK3/4 } & \multirow{2}{*}{$\begin{array}{l}\text { RK6 } \\
\text { Error }\end{array}$} & \multirow[b]{2}{*}{ NFE } \\
\hline Error & NFE & Error & NFE & Error & NFE & Error & NFE & & \\
\hline 7.37 & 20 & 4 & 12 & 3. & 20 & 3.6 & 20 & 1.6 & 8000 \\
\hline 66 & 401 & 6.8 & 2399 & 4.8 & 400 & 4.4 & 400 & 3.2 & 12000 \\
\hline .19 & 801 & 4 & 4786 & 5.9 & 700 & 5.7 & 700 & 5.2 & 24000 \\
\hline 11.41 & 1601 & 10.2 & 9550 & 7.0 & 1200 & 6.9 & 1200 & 6.8 & 45000 \\
\hline
\end{tabular}

and the EFRKN by Franco [15] are displayed in Table 3. The accuracy of the FFBNM with the exact solution and its efficiency in terms of number of function evaluation as compared with the aforementioned methods are displayed in Fig. 5 .

It is apparent from the data in Table 3 and the plots in Fig. 5 that the FFBNM is an efficient numerical integrator for this problem when compared with the TFARKN, EFRK and EFRKN methods.

\subsection{Example 4}

As a fourth test problem, we consider the Duffing equation

$$
y^{\prime \prime}+\left(\omega^{2}+\kappa^{2}\right) y=2 \kappa^{2} y^{3}, \quad y(0)=1, y^{\prime}(0)=\omega,
$$

whose solution in closed form is given by $y(t)=s n\left(\omega t ; \frac{k}{\omega}\right)$ and represents a periodic motion in terms of the Jacobian elliptic function $s n$. In our experiment, we take $\kappa=0.03$, and $\omega=5$ as the fitting parameter. This Problem was solved in the interval $[0,100]$ with step sizes $h=\frac{1}{2^{i}}$ for ETFFSH6S, a six stage explicit trigonometrically method of order seven by Li et al. [30], ARK4 and ARK3/8, both adapted RK methods of fourth order and four stages by Franco [15], and the RK6, the Butcher's sixth-order method given in Hairer et al. [19]. Table 4 shows the $-\log _{10}$ (MaxErr) of the numerical results. The accuracy of FFBNM in comparison with the exact results and the numerical results for $h=\frac{1}{2^{i}}, i=2,3,4,5$ with the above mentioned methods are presented in Fig. 6. 


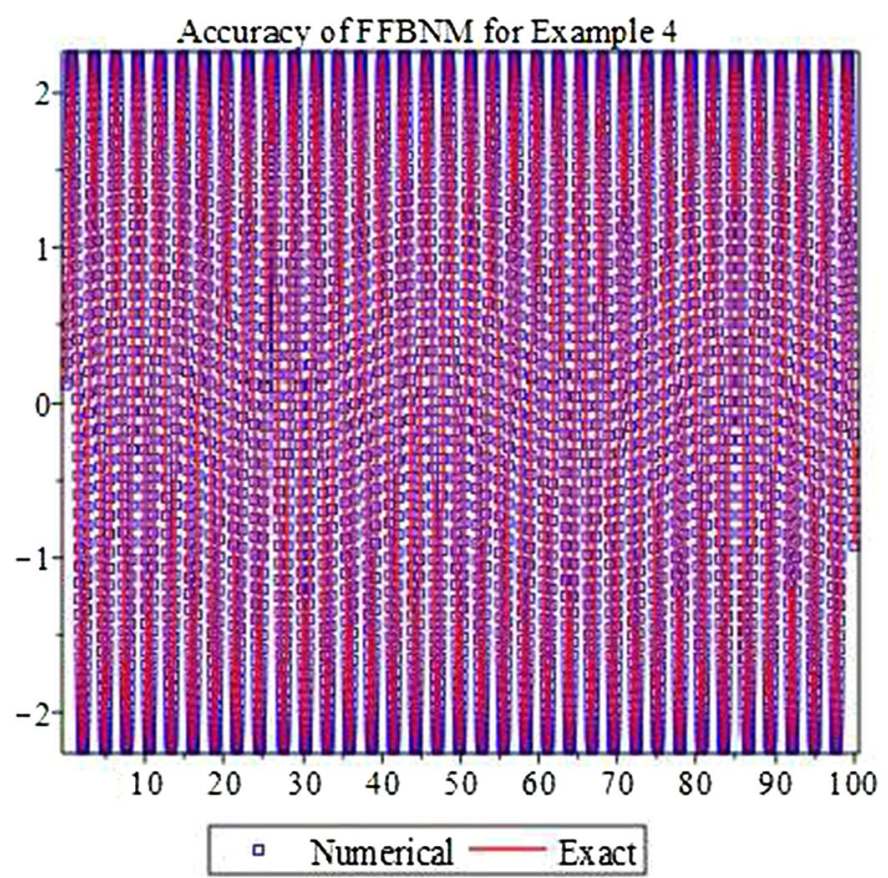

Efficiency Curve for Example 4

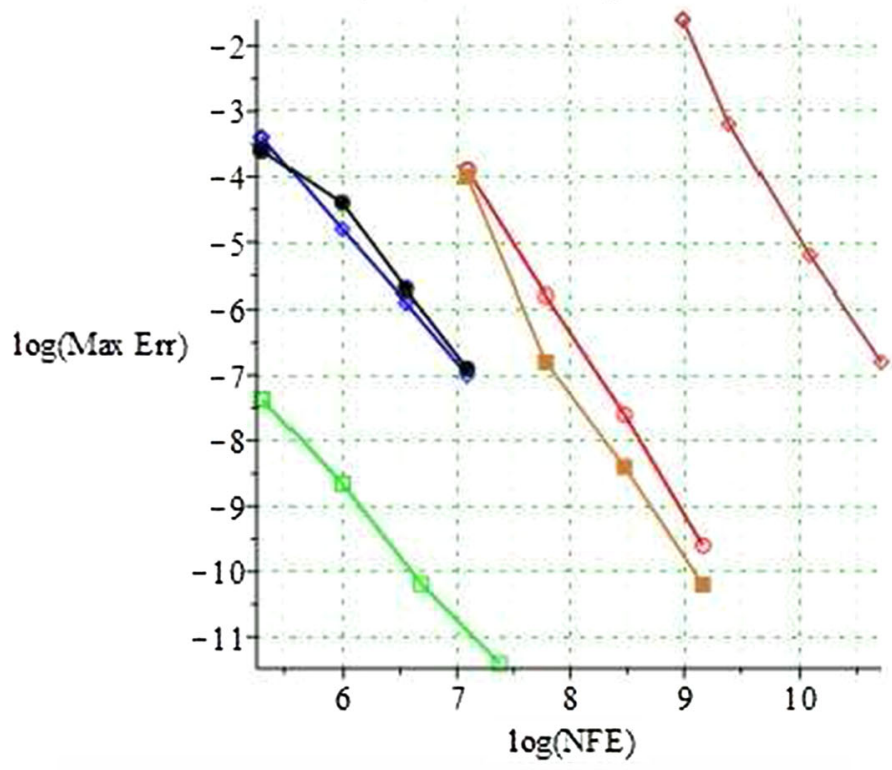

\begin{tabular}{|lllll}
\hline FFBNM & $\bullet$ ETFFSH5S & ETFFSH6S \\
$\diamond$ ARK4 & $\bullet$ ARK3/4 & $\diamond$ RK6 \\
\hline
\end{tabular}

Figure 6. The graphical representation of solution to Example 4 , and efficiency curves 
Table 5. Comparison of numerical results for example 5

\begin{tabular}{|c|c|c|c|c|c|}
\hline \multicolumn{2}{|c|}{ FFBNM } & \multicolumn{2}{|l|}{ BNM } & \multicolumn{2}{|l|}{$\mathrm{TFC}$} \\
\hline $\mathrm{N}$ & Error & $\mathrm{N}$ & Error & $\mathrm{N}$ & Error \\
\hline 1000 & $1.73 \times 10^{-3}$ & 1000 & $3.59 \times 10^{-3}$ & 5012 & $1.0 \times 10^{-6}$ \\
\hline 2000 & $1.44 \times 10^{-8}$ & 2000 & $6.31 \times 10^{-5}$ & 10000 & $1.3 \times 10^{-8}$ \\
\hline 4000 & $5.67 \times 10^{-11}$ & 4000 & $1.01 \times 10^{-6}$ & 17783 & $3.2 \times 10^{-9}$ \\
\hline 8000 & $9.90 \times 10^{-12}$ & 8000 & $1.60 \times 10^{-8}$ & 50119 & $1.0 \times 10^{-10}$ \\
\hline 16000 & $2.49 \times 10^{-12}$ & 16000 & $2.68 \times 10^{-10}$ & & \\
\hline
\end{tabular}

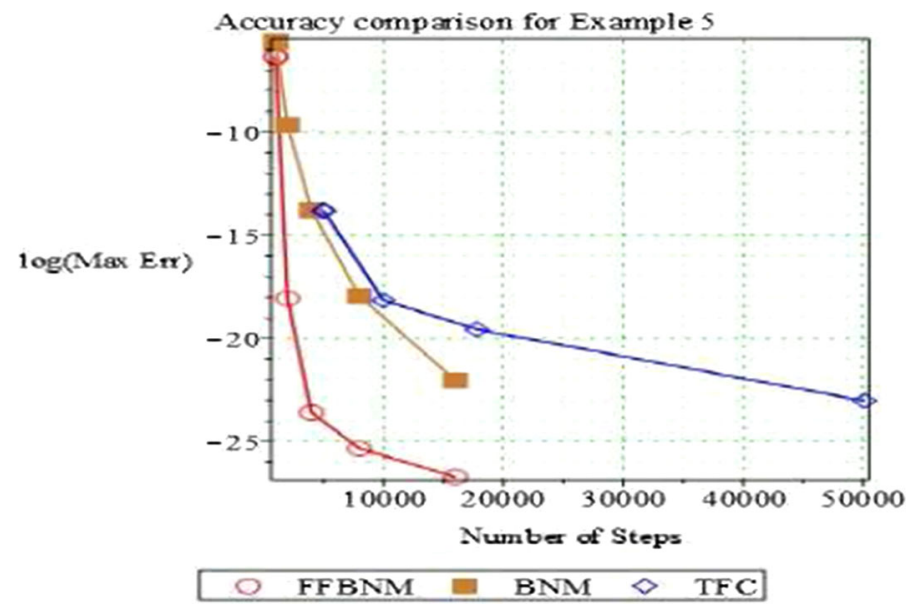

Figure 7. Efficiency curves for Example 5

\subsection{Example 5}

We consider the following perturbed two-body problem

$$
\left\{\begin{array}{c}
y_{1}^{\prime \prime}=-\frac{y_{1}}{r^{3}}-\frac{2\left(\epsilon+\epsilon^{2}\right) y_{1}}{r^{5}}, y_{1}(0)=1, y_{1}^{\prime}(0)=0, \\
y_{2}^{\prime \prime}=-\frac{y_{2}}{r^{3}}-\frac{2\left(\epsilon+\epsilon^{2}\right) y_{2}}{r^{5}}, y_{1}(0)=0, y_{1}^{\prime}(0)=1+\epsilon,
\end{array}\right.
$$

with $r=\sqrt{y_{1}^{2}+y_{2}^{2}}$, whose exact solution is given by $y_{1}=\cos (x+\epsilon x), y_{2}=$ $\sin (x+\epsilon x)$.

This system represents a motion on a perturbed circular orbit in the complex plane which occurs in classical mechanics and flexible body dynamics. The numerical results of the FFBNM on this problem with $\omega=1.01$ are compared with the Trigonometric Fourier Collocation (TFC) method by Wang [47] and the method BNM developed by Jator and Oladejo [23] on $[0,1000]$. The results given in Table 5 and the graphical representations in Fig. 7 taking $\epsilon=10^{-3}$, show that FFBNM is an accurate method for this perturbed Kepler's equation. 
Table 6. Comparison of numerical results for Example 6

\begin{tabular}{|c|c|c|c|c|c|c|c|}
\hline \multicolumn{2}{|c|}{ FFBNM } & \multicolumn{2}{|c|}{ ExtGauss2 } & \multicolumn{2}{|c|}{ Gauss3 } & \multicolumn{2}{|c|}{ ExtGauss3 } \\
\hline Error & $\mathrm{CPU}$ & Error & $\mathrm{CPU}$ & Error & $\mathrm{CPU}$ & Error & $\mathrm{CPU}$ \\
\hline 6.17 & 0.034 & 5.90 & 0.040 & 5.40 & 0.150 & 7.00 & 0.090 \\
\hline 8.21 & 0.070 & 7.10 & 0.050 & 7.10 & 0.290 & 9.40 & 0.140 \\
\hline 10.01 & 0.150 & 8.30 & 0.110 & 9.00 & 0.375 & 11.4 & 0.300 \\
\hline 11.87 & 0.370 & 9.50 & 0.210 & 10.60 & 0.730 & 12.00 & 0.600 \\
\hline
\end{tabular}

Table 7. Comparison of numerical results for Example 7

\begin{tabular}{|c|c|c|c|c|c|c|c|c|c|}
\hline \multicolumn{2}{|c|}{ FFBNM } & \multicolumn{2}{|l|}{$\mathrm{BNM}$} & \multicolumn{2}{|l|}{ AZD } & \multicolumn{2}{|l|}{ AME } & \multicolumn{2}{|l|}{ MAM } \\
\hline Error & NFE & Error & NFE & Error & $\mathrm{NFE}$ & Error & NFE & Error & NFE \\
\hline 65 & 101 & 2.18 & 101 & 25 & 1406 & 3.58 & 938 & 3.00 & 938 \\
\hline & 20 & & 20 & & 4688 & 4. & 3750 & 4. & 3750 \\
\hline . & 40 & 4 & 40 & 6.8 & 121 & 6.7 & 11250 & 6. & 9375 \\
\hline 12.24 & 801 & 5.32 & 801 & 8.41 & 39375 & 8.63 & 34688 & 8.29 & 30000 \\
\hline
\end{tabular}

\subsection{Example 6}

As our sixth experiment, we investigate the prevalent non-linear scalar Van der Pol equation given by

$$
y^{\prime \prime}+y=\delta\left(1-y^{2}\right) y^{\prime},
$$

with initial values

$$
y(0)=2+\frac{1}{96} \delta^{2}+\frac{1033}{552960} \delta^{4}+\frac{1019689}{55738368000} \delta^{2}, y^{\prime}(0)=0 .
$$

In our computations, we took the parameter $\delta$ as $\delta=10^{-3}$ and the fitting frequency as $\omega=1$. The integration of the problem was carried out in the interval $[0,100]$. For the comparison of the error of different methods, we use step lengths $h=\frac{1}{2^{i}}, i=1,2,3,4$. Since the analytic solution of this problem does not exists, we used a reference numerical solution which was obtained by Anderson and Geer [2] and Verhulst [45]. The Maximum global error, $-\log _{10}\left(\left\|y(x)-y_{n}\right\|_{\infty}\right)$, of the FFBNM compared with the Gauss methods studied in [49] are displayed in Table 6, while the efficiency curves are shown in Fig. 8, respectively. Whereas from the Table 6 and Fig. 8 it is evident that the FFBNM outperformed the ExtGauss2 of the same order and Gauss3 of order six, it competes favourably well with the ExtGauss3 of order six. In fact, the efficiency in terms of computational time of the FFBNM is almost half the computational time of the ExtGauss3. 


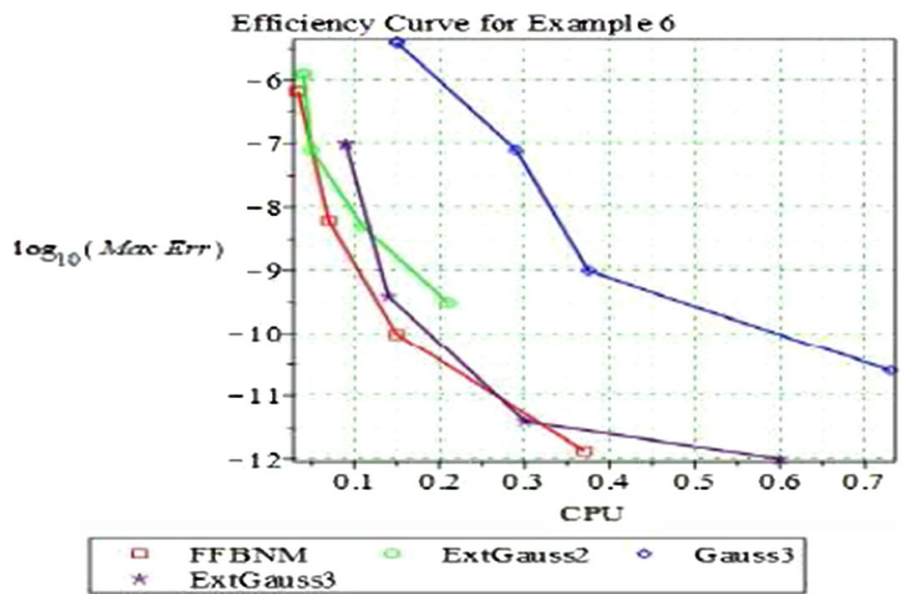

Figure 8. Efficiency curves for Example 6

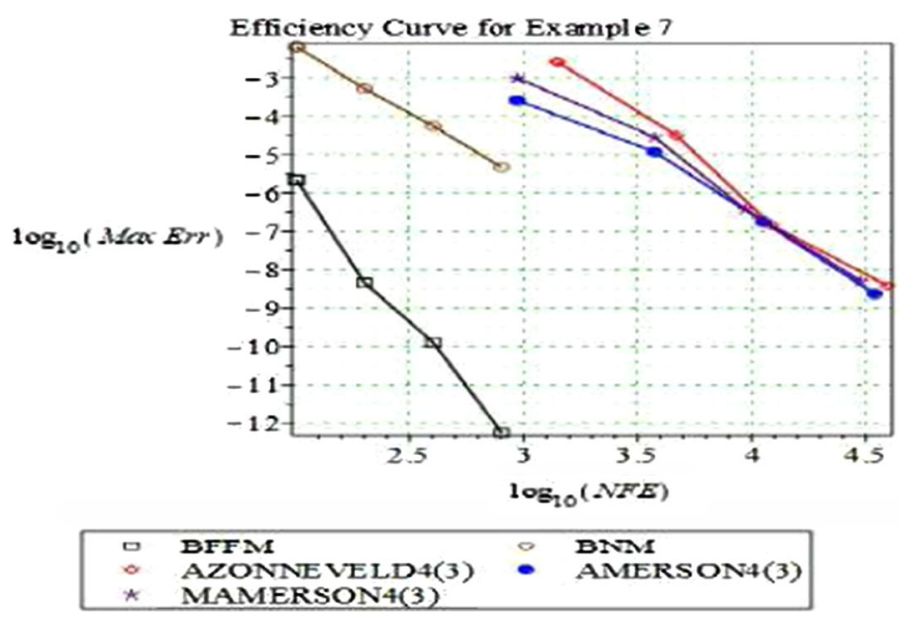

Figure 9. Efficiency curves for Example 7

\subsection{Example 7}

As our last example, we investigate the linear periodic problem studied in $[10,16]$

$$
y^{\prime \prime}+\omega y=\left((\omega)^{2}-4 x^{2}\right) \cos \left(x^{2}\right)-2 \sin \left(x^{2}\right), y(0)=1, y^{\prime}(0)=\omega
$$

whose solution in closed form $y(x)=\cos \left(x^{2}\right)+\sin (\omega x)$ represents a periodic motion that involves a constant frequency and a variable frequency.

For this problem, we integrate in the interval [0,5], taking the principal frequency as $\omega=50$. The numerical results stated in the Table 7 and Fig. 9 have been computed with the step lengths $h=\frac{0.1}{2^{i}}, i=1,2,3,4$. As can be seen in Table 7 and Fig. 9, the BNM, which is the limit method of 
the FFBNM, is not as accurate as the other fourth order methods AZONNEVELD4(3), AMERSON4(3)and MAMERSON4(3) (abbreviated AZD, AME and MAM respectively, for easy readability) presented in [16] but uses fewer number of function evaluations. However, the FFBNM performs better than its limiting method and some of the existing methods in the literature.

\section{Conclusions}

The numerical solution of oscillatory problems finds its best performance in the use of adapted methods. This article has developed a trigonometrically adapted method of Numerov type. Their characteristics have been studied, and some numerical examples have been presented, comparing the results of the proposed method with other methods appeared in the literature. These results allow us to conclude that the proposed method is competitive to solve problems whose solutions are oscillatory.

\section{Acknowledgements}

The authors would like to thank the anonymous reviewers for their constructive comments that greatly contributed to improve the article.

Funding Open Access funding provided thanks to the CRUE-CSIC agreement with Springer Nature.

Code availability Not applicable.

\section{Declarations}

Conflict of interest The authors declare no conflict of interest.

Open Access. This article is licensed under a Creative Commons Attribution 4.0 International License, which permits use, sharing, adaptation, distribution and reproduction in any medium or format, as long as you give appropriate credit to the original author(s) and the source, provide a link to the Creative Commons licence, and indicate if changes were made. The images or other third party material in this article are included in the article's Creative Commons licence, unless indicated otherwise in a credit line to the material. If material is not included in the article's Creative Commons licence and your intended use is not permitted by statutory regulation or exceeds the permitted use, you will need to obtain permission directly from the copyright holder. To view a copy of this licence, visit http:// creativecommons.org/licenses/by/4.0/.

Publisher's Note Springer Nature remains neutral with regard to jurisdictional claims in published maps and institutional affiliations. 


\section{Appendix: Specification of entries of The Lower Triangular Matrix $\varsigma$, The Upper Triangular Matrix $\Omega$, and Matrix $V$}

$$
\varsigma=\left[\begin{array}{ccccc}
1 & 0 & 0 & 0 & 0 \\
\frac{\sin \left(\omega x_{n+1}\right)}{\sin \left(\omega x_{n}\right)} & 1 & 0 & 0 & 0 \\
-\omega^{2} & 0 & 1 & 0 & 0 \\
-\frac{\omega^{2} \sin \left(\omega x_{n+1}\right)}{\sin \left(\omega x_{n}\right)} & -\omega^{2} & \frac{\sinh \left(\omega x_{n+1}\right)}{\sinh \left(\omega x_{n}\right)} & 1 & 0 \\
-\frac{\omega^{2} \sin \left(\omega x_{n+2}\right)}{\sin \left(\omega x_{n}\right)} & -\frac{\omega^{2} \sin \left(\omega x_{n}-\omega x_{n+2}\right)}{\sin \left(\omega x_{n}-\omega x_{n+1}\right)} & \varsigma_{53} & \varsigma_{54} & 1
\end{array}\right]
$$

with

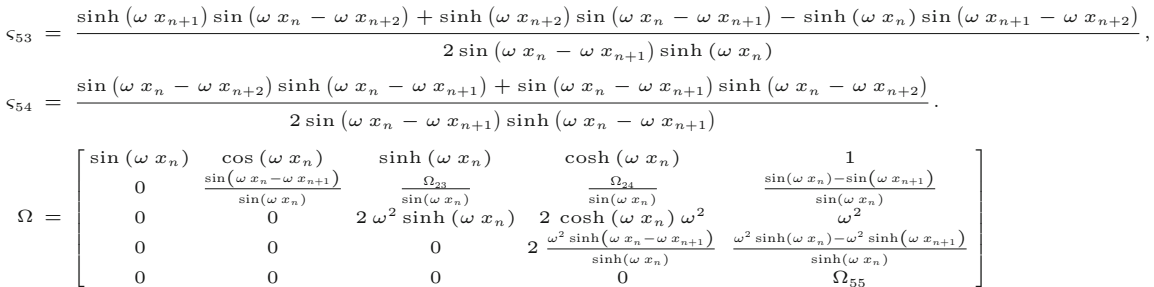

with

$$
\begin{aligned}
& \Omega_{23}=\sinh \left(\omega x_{n+1}\right) \sin \left(\omega x_{n}\right)-\sin \left(\omega x_{n+1}\right) \sinh \left(\omega x_{n}\right), \\
& \Omega_{24}=\cosh \left(\omega x_{n+1}\right) \sin \left(\omega x_{n}\right)-\sin \left(\omega x_{n+1}\right) \cosh \left(\omega x_{n}\right), \\
& \Omega_{55}=\frac{A}{2 \sin \left(\omega\left(x_{n}-x_{n+1}\right)\right) \sinh \left(\omega\left(x_{n}-x_{n+1}\right)\right)}
\end{aligned}
$$

where

$$
\begin{aligned}
A= & \omega^{2}\left(\sin \left(\omega\left(x_{n}-x_{n+2}\right)\right) \sinh \left(\omega\left(x_{n}-x_{n+1}\right)\right)-\sin \left(\omega\left(x_{n}-x_{n+1}\right)\right) \sinh \left(\omega\left(x_{n}-x_{n+2}\right)\right)\right. \\
& \left.+\sin \left(\omega\left(x_{n}-x_{n+1}\right)\right) \sinh \left(\omega\left(x_{n+1}-x_{n+2}\right)\right)-\sin \left(\omega\left(x_{n+1}-x_{n+2}\right)\right) \sinh \left(\omega\left(x_{n}-x_{n+1}\right)\right)\right) . \\
V= & {\left[\begin{array}{ccccc}
1 & \sin \left(\omega x_{n}\right) & \cos \left(\omega x_{n}\right) & \sinh \left(\omega x_{n}\right) & \cosh \left(\omega x_{n}\right) \\
1 & \sin \left(\omega x_{n+1}\right) & \cos \left(\omega x_{n+1}\right) & \sinh \left(\omega x_{n+1}\right) & \cosh \left(\omega x_{n+1}\right) \\
0 & -\omega^{2} \sin \left(\omega x_{n}\right) & -\omega^{2} \cos \left(\omega x_{n}\right) & \omega^{2} \sinh \left(\omega x_{n}\right) & \cosh \left(\omega x_{n}\right) \omega^{2} \\
0 & -\omega^{2} \sin \left(\omega x_{n+1}\right) & -\omega^{2} \cos \left(\omega x_{n+1}\right) & \omega^{2} \sinh \left(\omega x_{n+1}\right) & \cosh \left(\omega x_{n+1}\right) \omega^{2} \\
0 & -\omega^{2} \sin \left(\omega x_{n+2}\right) & -\omega^{2} \cos \left(\omega x_{n+2}\right) & \omega^{2} \sinh \left(\omega x_{n+2}\right) & \cosh \left(\omega x_{n+2}\right) \omega^{2}
\end{array}\right] . }
\end{aligned}
$$

\section{References}

[1] Abdulganiy, R.I., Akinfenwa, O.A., Okunuga, S.A.: Maximal order block trigonometrically fitted scheme for the numerical treatment of second order initial value problem with oscillating solutions. Int. J. Math. Anal. Optim. 2017, 168-186 (2018)

[2] Andersen, C.M., Geer, J.F.: Power series expansions for the frequency and period of the limit cycle of the Van Der Pol equation. SIAM J. Appl. Math. 42(3), 678-693 (1982)

[3] Archar, S.D.: Symmetric multistep Obrechkoff methods with zero phase-lag for periodic initial value problems of second order differential equations. J. Appl. Math. Comput. 218, 2237-2248 (2011)

[4] Awoyemi, D.O.: A P-stable linear multistep method for solving general third order ordinary differential equations. Int. J. Comput. Math. 80(8), 987-993 (2003) 
[5] Brugnano, L., Trigiante, D.: Solving Differential Problems by Multistep Initial and Boundary Value Methods. Gordan and Breach, Amsterdam (1998)

[6] Coleman, J.P., Duxbury, S.C.: Mixed collocation methods for $y^{\prime \prime}=f(x, y)$. J. Comput. Appl. Math. 126, 47-75 (2000)

[7] Enright, W.H.: Second derivative multistep method for stiff ODEs. SIAM J. Numer. Anal. 11(2), 321-331 (1974)

[8] Fang, Y., Wu, X.: A trigonometrically fitted explicit Numerov-type method for second order initial value problems with oscillating solutions. Appl. Numer. Math. 58, 341-351 (2008)

[9] Fang, Y., Song, Y., Wu, X.: A robust trigonometrically fitted embedded pair for perturbed oscillators. J. Comput. Appl. Math. 225, 347-355 (2009)

[10] Fang, Y.L., Yang, Y.P., You, X.: Revised trigonometrically fitted two step hybrid methods with equation dependent coefficients for highly oscillatory problems. J. Comput. Appl. Math. 318, 266-278 (2017)

[11] Fang, Y.L., Liu, C.Y., Wang, B.: Efficient energy-preserving methods for general nonlinear oscillatory hamiltonian system. Act. Math. Sin. 34, 1863-1878 (2018)

[12] Fatunla, S.O.: Numerical Methods for Initial Value Problems in Ordinary Differential Equations. Academic Press Inc., Cambridge (1988)

[13] Fatunla, S.O.: Block methods for second order ODEs. Int. J. Comput. Math. 41, 55-63 (1991)

[14] Franco, J.M.: An embedded pair of Exponentially-Fitted explicit Runge-Kutta methods. J. Comput. Appl. Math. 149, 407-414 (2002)

[15] Franco, J.M.: Runge-Kutta-methods adapted to the numerical integration of oscillatory problems. Appl. Numer. Math. 50, 427-443 (2004)

[16] Franco, J.M.: Runge-Kutta methods adapted to the numerical integration of oscillatory problems. Appl. Numer. Math. 50, 427-443 (2004)

[17] Franco, J.M.: A class of explicit two-step hybrid methods for second-order IVPs. J. Comput. Appl. Math. 187, 41-57 (2006)

[18] Hairer, E., Wanner, G.: A theory for Nyström methods. Numerische Mathematik 25, 383-400 (1976)

[19] Hairer, E., Nörsett, S.P., Wanner, G.: Solving Ordinary Differential Equations I. Nonstiff Problems. Springer, Berlin (1993)

[20] Jain, M.K., Aziz, T.: Cubic spline solution of two-point boundary value problems with significant first derivatives. Comput. Methods Appl. Mech. Eng. 39, 83-91 (1983)

[21] Jator, S.N.: Solving second order initial value problems by a hybrid multistep method without predictors. Appl. Math. Comput. 277, 4036-4046 (2010)

[22] Jator, S.N.: Implicit third derivative Runge-Kutta-Nyström method with trigonometric coefficients. Numer. Algorithms 70(1), 133-150 (2015). https:// doi.org/10.1007/s11075-014-9938-5

[23] Jator, S.N.: Block third derivative method based on trigonometric polynomials for periodic initial-value problems. Afrika Matematika 27, 365-377 (2016)

[24] Jator, S.N., Li, J.: A self-starting linear multistep method for a direct solution of the general second order initial value problem Intern. J. Comput. Math. 86, 827-836 (2009) 
[25] Jator, S.N., Oladejo, H.B.: Block Nyström method for singular differential equations of the Lane-Emdem Type and problems with highly oscillatory solutions. Int. J. Appl. Comput. Math. 3, 1385-1402 (2017). https://doi.org/10. 1007/s40819-017-0425-2

[26] Jator, S.N., Swindle, S., French, R.: Trigonometrically fitted block Numerov type method for $y^{\prime \prime}=f\left(x, y, y^{\prime}\right)$. Numer. Algorithms 62, 13-26 (2013)

[27] Jator, S.N., Akinfenwa, A.O., Okunuga, S.A., Sofoluwe, A.B.: High-order continuous third derivative formulas with block extension for $y^{\prime \prime}=f\left(x, y, y^{\prime}\right)$. Int. J. Comput. Math. 90(9), 1899-1914 (2013)

[28] Lambert, J.D.: Computational Methods in Ordinary Differential System, The Initial Value Problem. Wiley, New York (1973)

[29] Lambert, J.D., Watson, I.A.: Symmetric multistep methods for periodic initial value problems. J. Inst. Math. Appl. 18, 189-202 (1976)

[30] Li, J., Lu, M., Qi, X.: Trigonometrically fitted multi-step hybrid methods for oscillatory special second-order initial value problems. Int. J. Comput. Math. 95, 979-997 (2018). https://doi.org/10.1080/00207160.2017.1303138

[31] Onumanyi, P., Awoyemi, D.O., Jator, S.N., Sirisena, U.W.: New linear mutlistep methods with continuous coefficients for first order initial value problems. J. Nig. Math. Soc. 13, 37-51 (1994)

[32] Ramos, H., Lorenzo, C.: Review of explicit Falkner methods and its modifications for solving special second-order I.V.P.s. Comput. Phys. Commun. 181(11), 1833-1841 (2010)

[33] Ramos, H., Patricio, M.F.: Some new implicit two-step multiderivative methods for solving special second-order IVP's. Appl. Math. Comput. 239, 227-241 (2014)

[34] Ramos, H., Rufai, M.A.: Third derivative modification of $k$-step block Falkner methods for the numerical solution of second order initial-value problems. Appl. Math. Comput. 333, 231-245 (2018)

[35] Ramos, H., Vigo-Aguiar, J.: Variable-stepsize Chebyshev-type methods for the integration of second-order I.V.P.'s. J. Comput. Appl. Math. 204(1), 102-113 (2007)

[36] Ramos, H., Vigo-Aguiar, J.: A trigonometrically-fitted method with two frequencies, one for the solution and another one for the derivative. Comput. Phys. Commun. 185, 1230-1236 (2014)

[37] Ramos, H., Singh, G., Kanwar, V., Bhatia, S.: An efficient variable step-size rational Falkner-type method for solving the special second-order IVP. Appl. Math. Comput. 291, 39-51 (2016)

[38] Ramos, H., Mehta, S., Vigo-Aguiar, J.: A unified approach for the development of $k$-step block Falkner-type methods for solving general second-order initialvalue problems in ODEs. J. Comput. Appl. Math. 318, 550-564 (2017)

[39] Simos, T.E.: A P-stable complete in Phase Obrechkoff trigonometrically fitted method for periodic initial value problems. Proc. R. Soc. 441, 283-289 (1993)

[40] Singh, G., Ramos, H.: An optimized two-step hybrid block method formulated in variable step-size mode for integrating $y^{\prime \prime}=f\left(x, y, y^{\prime}\right)$ numerically. Numer. Math. Theor. Meth. Appl. 12, 640-660 (2019)

[41] Tsitouras, Ch.: Explicit eight order two step methods with nine stages for integrating oscillatory problems. Int. J. Mod. Phys. 17, 861-876 (2006) 
[42] Twizel, E.H., Khaliq, A.Q.M.: Multiderivative methods for periodic IVPs SIAM. J. Numer. Anal. 21, 111-121 (1984)

[43] van Daele, M., Vanden Berghe, G.: P-stable exponentially fitted Obrechkoff methods of arbitrary order for second order differential equations. Numer. Algor. 46, 333-350 (2007)

[44] van Dooren, R.: Stabilization of Cowell's classical finite difference methods for numerical integration. J. Comput. Physi. 16, 186-192 (1974)

[45] Verhulst, F.: Nonlinear Differential Equations and Dynamical Systems. Springer, Berlin (1990)

[46] Wang, Z., Zhao, D., Dai, Y., Wu, D.: An improved trigonometrically fitted p-stable Obrechkoff method for periodic initial value problems. Proc. R. Soc. 461, 1639-1658 (2005)

[47] Wang, B., Iserles, A., Wu, X.: Arbitrary-order trigonometric fourier collocation methods for multi-frequency oscillatory systems. Found. Computat. Math. 16(1), 151-181 (2015). https://doi.org/10.1007/s10208-014-9241-9

[48] Wu, X., You, X., Wang, B.: Structure-Preserving Algorithms for Oscillatory Differential Equations. Springer, Berlin (2013)

[49] You, X., Zhang, R., Huang, T., Fang, Y.: Symmetric collocation ERKN methods for general second-order oscillators. Calcolo 52, 122 (2019)

R. I. Abdulganiy

Distance Learning Institute

University of Lagos

Lagos

Nigeria

e-mail: profabdulcalculus@gmail.com

Higinio Ramos

Department of Applied Mathematics

University of Salamanca

Salamanca

Spain

e-mail: higra@usal.es

O. A. Akinfenwa and S. A. Okunuga

Department of Expunge Applied Mathematics

University of Lagos

Lagos

Nigeria

e-mail: akinolu35@yahoo.com

S. A. Okunuga

e-mail: sokunuga@unilag.edu.ng

Received: May 19, 2020.

Revised: January 15, 2021.

Accepted: October 1, 2021. 